Draft Version September 12, 2018

Preprint typeset using $\mathrm{IAT}_{\mathrm{E}} \mathrm{X}$ style emulateapj v. 5/2/11

\title{
CONSTRAINING THE X-RAY AND COSMIC RAY IONIZATION CHEMISTRY OF THE TW HYA PROTOPLANETARY DISK: EVIDENCE FOR A SUB-INTERSTELLAR COSMIC RAY RATE
}

\author{
L. Ilsedore Cleeves ${ }^{1}$, Edwin A. Bergin ${ }^{1}$, Chunhua QI $^{2}$, Fred C. Adams $^{1,3}$, Karin I. ÖBerg ${ }^{2}$ \\ Draft version September 12, 2018
}

\begin{abstract}
We present an observational and theoretical study of the primary ionizing agents (cosmic rays and $\mathrm{X}$-rays) in the TW Hya protoplanetary disk. We use a set of resolved and unresolved observations of molecular ions and other molecular species, encompassing eleven lines total, in concert with a grid of disk chemistry models. The molecular ion constraints comprise new data from the Submillimeter Array on $\mathrm{HCO}^{+}$, acquired at unprecedented spatial resolution, and data from the literature, including ALMA observations of $\mathrm{N}_{2} \mathrm{H}^{+}$. We vary the model incident CR flux and stellar X-ray spectra and find that TW Hya's $\mathrm{HCO}^{+}$and $\mathrm{N}_{2} \mathrm{H}^{+}$emission are best fit by a moderately hard X-ray spectra, as would be expected during the "flaring" state of the star, and a low CR ionization rate, $\zeta_{\mathrm{CR}} \lesssim 10^{-19} \mathrm{~s}^{-1}$. This low $\mathrm{CR}$ rate is the first indication of the presence of $\mathrm{CR}$ exclusion by winds and/or magnetic fields in an actively accreting $\mathrm{T}$ Tauri disk system. With this new constraint, our best fit ionization structure predicts a low turbulence "dead-zone" extending from the inner edge of the disk out to $50-65 \mathrm{AU}$. This region coincides with an observed concentration of millimeter grains, and we propose that the inner region of TW Hya is a dust (and possibly planet) growth factory as predicted by previous
\end{abstract} theoretical work.

Subject headings: accretion, accretion disks — astrochemistry — circumstellar matter — stars: premain sequence

\section{INTRODUCTION}

Gas-rich circumstellar disks around young stars are the formation sites of planetary systems. The physical conditions of this circumstellar material, including density, temperature, and ionization, all play an important role in setting the dynamical and chemical properties of the disk. In particular, ionization has a central role in governing disk turbulence and chemistry within the cold $(T<100 \mathrm{~K})$ planet-forming gas. The turbulence of disks with masses comparable to our own minimum mass solar nebula $\left(\lesssim 0.05 \mathrm{M}_{\odot}\right.$; Weidenschilling 1977) is thought to be driven by the magnetorotational instability (MRI; e.g., Velikhov 1959; Balbus \& Hawley 1991; Stone et al. 1996, Wardle 1999 , Sano et al. 2000, Sano \& Stone 2002; Fleming \& Stone 2003 Bai \& Stone 2011). MRI requires the disk to be sufficiently ionized such that the bulk, predominantly neutral gas can couple to the magnetic field lines, thereby "stirring" the gas. Regions of the disk quiescent to such turbulence, i.e., "dead-zones," have been posited as safe-havens for efficient planetesimal formation (Gressel et al. 2012), as well as an efficient "stopping mechanism" against Type I and II migration (e.g., Matsumura \& Pudritz 2005, Matsumura et al. 2007). With regards to molecular composition, ionization drives the most efficient chemical processes in the cold, dense regions of disks, both in the gas by ion-neutral chemical pathways (Herbst \& Klemperer 1973) and through ionization-derived hydrogenation reactions on ice-coated grain surfaces (Tielens \& Hagen 1982, Hasegawa et al.

\footnotetext{
${ }^{1}$ Department of Astronomy, University of Michigan, 1085 S. University Ave., Ann Arbor, MI 48109

${ }^{2}$ Harvard-Smithsonian Center for Astrophysics, 60 Garden Street, Cambridge, MA 02138

${ }^{2}$ Department of Physics, University of Michigan, 450 Church St, Ann Arbor, MI 48109
}

1992: Garrod et al. 2008). For the same reason, ionization plays a pivotal role in facilitating (or hindering) deuterium fractionation reactions in the gas or on cold grain surfaces (Aikawa \& Herbst 1999. Cleeves et al. 2014b). Consequently, ionization is central to the chemical and physical fate of protoplanetary disks and ultimately the planets they form.

The primary sources of dense gas ionization in disks are $\mathrm{X}$-rays, cosmic rays (CRs) and the decay of short-lived radionuclides (SLRs). Classical T Tauri (CTT) stars are exceptionally X-ray bright $\left(10^{28} \mathrm{erg} \mathrm{s}^{-1} \mathrm{~cm}^{-2} \lesssim L_{\mathrm{XR}} \lesssim\right.$ $10^{34} \mathrm{erg} \mathrm{s}^{-1} \mathrm{~cm}^{-2}$; Feigelson et al. 2002) and often timevariable sources. X-ray flaring activity in CTTs is commonly associated with an overall hardening of the Xray spectrum, where relatively more energy is output at $E_{\mathrm{XR}} \gtrsim 2 \mathrm{keV}$ (Skinner et al. 1997). These harder X-ray photons are particularly important in setting the disk ionization as they are not easily impeded by intervening material between the star and the disk (i.e., by a stellar/disk wind) and are not as efficiently stopped within the disk itself compared to less-energetic $E_{\mathrm{XR}} \sim 1 \mathrm{keV}$ photons (Glassgold et al. 1997).

In very dense gas $\left(n_{\mathrm{H}_{2}} \gtrsim 10^{9} \mathrm{~cm}^{-3}\right)$, where X-rays are strongly attenuated, the primary sources of ionization available are external galactic CRs and the internal decay of short-lived radionuclides (SLRs). In the dense interstellar medium, CRs ionize at a rate exceeding a few times $10^{-17} \mathrm{~s}^{-1}$ and perhaps an order of magnitude or more higher in the diffuse gas (Indriolo \& McCall 2012). However, in the presence of stellar winds and/or magnetic fields, the incident $\mathrm{CR}$ rate can be substantially reduced by orders of magnitude (Dolginov \& Stepinski 1994; Cleeves et al. 2013a; Padovani et al. 2013; Fatuzzo \& Adams 2014).

SLRs are provided by massive stars that enrich the 
dense molecular gas from which young stars (and disks) form. In addition, certain species including ${ }^{36} \mathrm{Cl}$ and, to some extent, ${ }^{26} \mathrm{Al}$ (e.g., Gounelle et al. 2001) can be provided by grain-surface spallation via energetic particles from the central star; however, the dominant species at $1 \mathrm{Myr},{ }^{26} \mathrm{Al}$, is primarily formed by external sources (see reviews by Adams 2010; Dauphas \& Chaussidon 2011. and references therein). The presence of SLRs in the young Solar Nebula is inferred from the Solar System's meteoritic record, but the contribution towards disk ionization from SLR decay is uncertain, owing to unknown initial abundances, inherent time-decay over the lifetime of the disk, and an uncertain "injection" point prior to or after disk formation, or perhaps both (e.g., Ouellette et al. 2007, 2010; Adams et al. 2014). SLR ionization may also be reduced by the escape of ionizing agents, i.e., the decay products, from the tenuous outer disk (Cleeves et al. 2013b).

All of these effects act together to make a rich ionization environment with substantial spatial variation. The individual importance of each of these physical processes has been debated for decades (Gammie 1996; Igea \& Glassgold 1999). For the gas thermal structure in the upper layers, understanding the heating contribution by $\mathrm{X}$-rays, UV irradiation and to a lesser extent the CR flux is necessary (e.g. Glassgold et al. 2004; Jonkheid et al. 2004 Kamp \& Dullemond 2004; Gorti \& Hollenbach 2009 Glassgold et al. 2012 Bruderer et al. 2012). In determining the extent of the disk that is unstable to MRI (i.e., is turbulent), the question is often what minimum ionization fraction is required. CRs, given their ability to penetrate disk gas down to $\lesssim 100 \mathrm{~g} \mathrm{~cm}^{-2}$, were the original focus of Gammie (1996) in determining the thickness of the MRI active layer. However, given the intensity of the young star as an X-ray source and including the important effects of X-ray scattering, Igea \& Glassgold (1999) argued that the disk could be turbulent everywhere beyond $5 \mathrm{AU}$ (inside of which X-rays are too highly attenuated) even in the absence of CRs. With the arrival of spatially and spectrally resolved high signalto-noise data on molecular ions, many of these questions should be possible to resolve through the coupling of detailed models and observations.

In Cleeves et al. (2014a), we explored the sensitivity of disk ion chemistry to different ionization scenarios using a generic $\mathrm{T}$ Tauri disk model. In the present paper, we apply these results to a particular protoplanetary disk, TW Hya. TW Hya's proximity $(d=55 \pm 9$ pc; Webb et al. 1999: van Leeuwen 2007), face-on inclination $\left(i \sim 7 \pm 1^{\circ}\right.$; Qi et al. 2004), and general isolation from ambient molecular gas (Rucinski \& Krautter 1983 Feigelson 1996: Hoff et al. 1998; Tachihara et al. 2009), together provide a clear view into the chemical and physical properties of TW Hya's circumstellar material. This favorable orientation combined with a rich observed gasphase chemistry $\left(\mathrm{CN}, \mathrm{HCN}, \mathrm{DCN}, \mathrm{H}_{2} \mathrm{O}, \mathrm{HD}\right.$, and $\mathrm{H}_{2} \mathrm{CO}$; Kastner et al. 1997; van Zadelhoff et al. 2001; Qi et al. 2008 Hogerheijde et al. 2011: Oberg et al. 2012; Bergin et al. 2013: Qi et al. 2013a) and numerous detections of molecular ion emission $\left(\mathrm{N}_{2} \mathrm{H}^{+} \mathrm{HCO}^{+}, \mathrm{H}^{13} \mathrm{CO}^{+}\right.$, and $\mathrm{DCO}^{+}$; Kastner et al. 1997; van Zadelhoff et al. 2001; Wilner et al. |2003 van Dishoeck et al. 2003; Qi et al. 2008 2013a) make for fertile ground to study the disk's ionization chemistry. In this work, we combine new and archival data with detailed theoretical simulations of disk ionization chemistry to unravel the origin of the molecular ion emission along with its distribution within the TW Hya protoplanetary disk.

In \$2, we present the observational constraints, including new data from the Submillimeter Array (SMA). We describe the gas and dust physical model along with the chemical code in $\$ 3$. In 33.3 we outline the grid of ionization parameters, where we specifically vary the shape of the incident X-ray spectrum and incident CR ionization rate. We calculate chemical models across the grid and perform simulated observations $(\$ 3.4$ ) for direct comparison to the data. We present our findings in 4 , discuss their implications for the TW Hya disk structure in $\$ 5$ and summarize in $\$ 6$.

\section{OBSERVATIONS}

\subsection{Submillimeter Array Data}

The $\mathrm{HCO}^{+}(3-2)$ observations of TW Hydra were made with the SMA (Ho et al. 2004) located atop Mauna Kea on April 12, 2012 in the very extended (VEX) configuration with 7 antennas under excellent sky conditions, $\tau_{225 \mathrm{GHz}} \lesssim 0.04$. Titan was used for flux calibration, the quasars J1147-382 and J1037-295 were used for gain calibration and 3C279 for passband calibration. The line was observed with 256 channels in chunk s04, where the chunk width is $104 \mathrm{MHz}$, corresponding to a velocity resolution of $\Delta v=0.46 \mathrm{~km} \mathrm{~s}^{-1}$. All data were phase- and amplitude-calibrated using the MIR software packag $\AA^{4}$ All continuum and spectral line maps were then generated and CLEANed using the MIRIAD software package with natural weighting. The synthesized VEX beam for the $\mathrm{HCO}^{+}$observation is $\left(\theta_{\text {maj }} \times \theta_{\text {min }}\right)=\left(0.64^{\prime \prime} \times 0.36^{\prime \prime}\right)$ with a position angle $\mathrm{PA}=17.3^{\circ}$, where the RMS noise on the line is $85 \mathrm{mJy} /$ beam. We have also combined the new VEX $\mathrm{HCO}^{+}$track with shorter spacing data from compact and extended tracks (Qi et al. 2008, observed between 2005-2006) to improve the imaging fidelity. The synthesized beam for the combined measurement set is $0.69^{\prime \prime} \times 0.39^{\prime \prime}, \mathrm{PA}=16.9^{\circ}$, with an RMS of $60 \mathrm{mJy} /$ beam. The $1 \sigma$ continuum sensitivity at 267 $\mathrm{GHz}$ is $1.6 \mathrm{mJy}$ beam $^{-1}\left(0.63^{\prime \prime} \times 0.35^{\prime \prime}\right.$ beam $)$ and 2.3 mJy beam ${ }^{-1}\left(0.65^{\prime \prime} \times 0.37^{\prime \prime}\right.$ beam $)$ beam in the VEX-only and combined observations, respectively. The decrease in sensitivity in the latter is a result of poorer atmospheric conditions for the extended and compact tracks. Figure 1 shows the velocity integrated line flux for the combined and VEX-only data sets.

Observations of the $\mathrm{H}^{13} \mathrm{CO}^{+}(3-2)$ line were made on 2014 April 8 using six out of eight 6-m antennas of the SMA in the extended configuration with projected baselines ranging from 8 to 165 meters. The tuning was centered on the $\mathrm{H}^{13} \mathrm{CO}^{+}(3-2)$ line at $260.255339 \mathrm{GHz}$ in chunk S23. The observing loops used J1037-295 as the gain calibrator. The bandpass response was calibrated using observations of $3 \mathrm{C} 279$. Flux calibration was done using observations of Titan and Callisto. The derived flux of J1037-295 at the time of the observations was $0.70 \mathrm{Jy}$. The spatially integrated $\mathrm{H}^{13} \mathrm{CO}^{+}(3-2)$ and $\mathrm{HCO}^{+}(3-2)$ spectra are shown in Figure 2 .

\footnotetext{
${ }^{4}$ http://www.cfa.harvard.edu/ cqi/mircook.html
} 

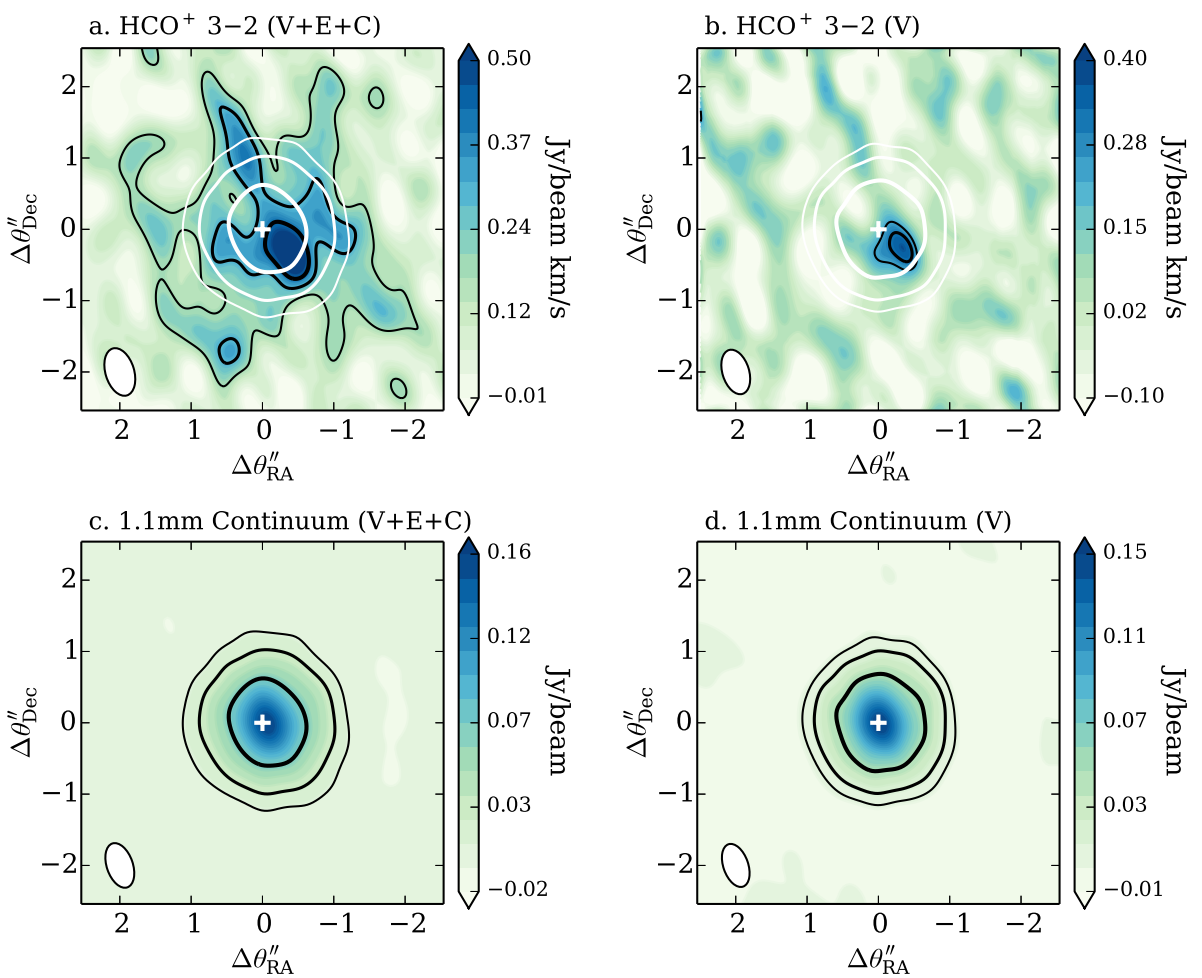

Figure 1. TW Hya velocity integrated $\mathrm{HCO}^{+}(3-2)(t o p)$ and $1.1 \mathrm{~mm}$ continuum (bottom) observations. The combined SMA veryextended, extended and compact array data in panels (a) and (c) show the almost symmetric large scale structure of the $\mathrm{HCO}^{+}$emission, while the very-extended data in panels (b) and (d) show the small-scale deviation from axis-symmetry in $\mathrm{HCO}^{+}$. White crosshairs mark the continuum phase center and overlaid white contours trace the continuum contours. Black contours mark $3 \sigma, 5 \sigma$ and $7 \sigma$ in the $\mathrm{HCO}^{+}$ panels with $1 \sigma=60 \mathrm{mJy}$ beam ${ }^{-1} \mathrm{~km} \mathrm{~s}^{-1}$ (left) and $85 \mathrm{mJy} \mathrm{beam}^{-1} \mathrm{~km} \mathrm{~s}^{-1}$ (right). Continuum contours are $3 \sigma, 10 \sigma$ and $30 \sigma$, where $1 \sigma=2.3 \mathrm{mJy} \mathrm{beam}^{-1}$ (left) and $1 \sigma=1.6 \mathrm{mJy}^{\text {beam }}{ }^{-1}$ (right). The beam is shown in the lower-left of each panel.

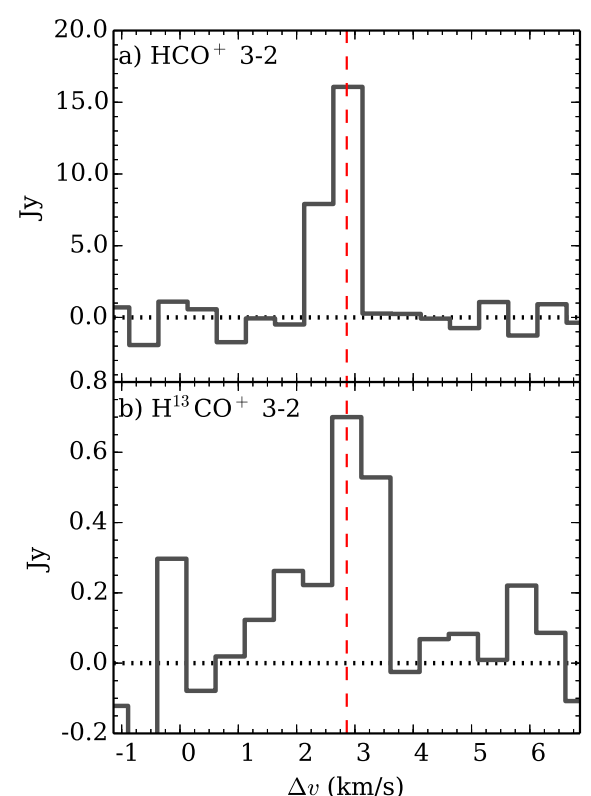

Figure 2. Spatially integrated spectra over an $8^{\prime \prime}$ region, where the vertical red line indicates TW Hya's intrinsic velocity $V_{\mathrm{LSR}}=2.86 \mathrm{~km} \mathrm{~s}^{-1}$. Top: $\mathrm{HCO}^{+}(3-2)$ from the combined $(\mathrm{V}+\mathrm{E}+\mathrm{C})$ data set. Bottom: $\mathrm{H}^{13} \mathrm{CO}^{+}(3-2)$ from the extended configuration observations.

\subsection{Archival Data}

In addition to the new $\mathrm{HCO}^{+}(3-2)$ and $\mathrm{H}^{13} \mathrm{CO}^{+}$ $(3-2)$ data, we have compiled molecular ion emission line observations from archives and the literature (Table 1). $\mathrm{HCO}^{+}(1-0)$ and $(4-3)$ line data was extracted from ALMA Science Verification observations. The $\mathrm{HCO}^{+}$ $(1-0)$ line was observed in Band 3 on May 13-14, 2011 with ten $12-\mathrm{m}$ antennas for a total of 3.7 hours. Titan, $3 \mathrm{C} 279$, and J1037-295 were used for flux, gain and phase calibration, respectively. The $\mathrm{HCO}^{+}(4-3)$ line was observed in Band 7 on April 22, 2011 in three scheduling blocks for a total of 4.5 hours. Nine $12-\mathrm{m}$ antennas were available during the observations; however, one antenna had to be flagged. The same calibrators were used as in the $\mathrm{HCO}^{+}(1-0)$ observations. Further information regarding the observations is provided at the ALMA Science Verification websit 5 . From the publicly available calibrated data, we extract the flux from an $8^{\prime \prime}$ region for both lines.

$\mathrm{H}^{13} \mathrm{CO}^{+}(4-3), \mathrm{N}_{2} \mathrm{H}^{+}(3-2)$ and $(4-3)$ have been observed with the SMA and ALMA, respectively (Qi et al. 2008, 2013a b). Integrated line fluxes were extracted from the spectral image cubes using an 8" box to be consistent with the new and archival $\mathrm{HCO}^{+}$data. In addition to these molecular line data, we have made use of the published HD, CO and HCN fluxes listed in in Table 1 to calibrate and verify the developed TW Hya disk chemistry model.

All reported noise in Table 1 combines in quadrature the random noise on the data and an absolute flux uncertainty of $15 \%$. We note that as a result the detections

\footnotetext{
${ }^{5}$ https://almascience.nrao.edu/alma-data/science-verification
} 
are individually more significant, i.e., have higher signal to noise as determined from random noise, than the table implies. See section \$3.4 for a detailed discussion regarding the "Recover All?" column.

\section{MODELING}

To evaluate what constraints the molecular ion observations provide on the ionization agents active in the TW Hya disk requires 1) a physical model of the TW Hya protoplanetary disk, 2) a disk chemistry code, and 3 ) the application of this code to the physical model under a range of ionizing conditions. Informed by previous model efforts (e.g., Calvet et al. 2002, Thi et al. 2010. Gorti et al. 2011; Andrews et al. 2012; Menu et al. 2014) and directly building on Bergin et al. (2013), we have constructed a new physical model of the TW Hya disk, which focuses on the aspects of most importance to the molecular ion chemistry, i.e. the distribution of cold gas and small dust grains. The details of the modeling process are described in Appendix A as well as the sensitivity of our conclusion on the chosen disk structure, and we review only the main features of the model here.

\subsection{Physical Structure}

The dust structure is derived from fitting the spectral energy distribution, where we include settling by defining two dust populations of small (atmosphere) and large (midplane) grains, with the latter concentrated near the midplane, i.e. it is parameterized as having a smaller scale height. The outer disk radius of our full model is taken to be $R_{\text {out }}=200 \mathrm{AU}$ as determined from the CO gas disk and scattered light images tracing the small dust (Andrews et al. 2012, Krist et al. 2000; Trilling et al. 2001; Weinberger et al. 2002; Roberge et al. 2005; Debes et al. 2013). We do not account for the observed radial variation between the two dust populations, i.e., the concentration of large grains inside of $R \sim 60$ AU (Andrews et al. 2012), since the explored chemistry depends mainly on the small dust grain population, which dominate the total surface area of grains (see 3.2 ).

We assume the gas is co-distributed with the small grains following a power-law with an exponential taper beyond $R>150$ AU (see Appendix C for structure dependence) and cut off at an outer disk radius of $200 \mathrm{AU}$. We estimate the total mass in gas from the Herschel detection of HD (Bergin et al. 2013), by varying the diskintegrated gas-to-dust mass ratio, i.e., the gas-to-dust ratio calculated from the column density is constant, but the local gas-to-dust ratio varies with height (due to settling). The resulting disk gas mass in our model is $M_{g}=0.04 \mathrm{M}_{\odot}$ with an uncertainty of $0.02 \mathrm{M}_{\odot}$ (see Appendix A). The gas temperatures are estimated from the UV radiation field throughout the disk ( $\mathrm{S}$. Bruderer, in private communication and Appendix A. The gas and dust distributions of our physical models are shown in Figure 3 .

\subsection{Disk Chemistry}

We employ a time-dependent chemical code specifically tailored to the disk chemical environment (Fogel et al. 2011). The code solves the input reaction network based on the input disk physical parameters and initial chemical abundances. The baseline chemical reaction network of Fogel et al. (2011) is built from the OSU gas-phase network (Smith et al. 2004) and includes neutral-neutral, ion-neutral, ion recombination with grains/electrons, freeze-out, thermal and non-thermal desorption via UV photons and CRs, photodissociation, photoionization, Xray induced $\mathrm{UV}$, self-shielding of $\mathrm{CO}$ and $\mathrm{H}_{2}$, and water and $\mathrm{H}_{2}$ grain-surface formation. The expanded network (Cleeves et al. 2014a) also includes simple deuterium reactions to form $\mathrm{H}_{2} \mathrm{D}^{+}$and $\mathrm{HDO}$, and self-shielding of $\mathrm{HD}$ and $\mathrm{D}_{2}$. The grain-surface formation reactions are also extended in the present work to include additional pathways for grain surface chemistry in the formalism of Hasegawa et al. (1992) for a small subset of species (77 reactions total), forming $\mathrm{H}_{2} / \mathrm{HD} / \mathrm{D}_{2}, \mathrm{H}_{2} \mathrm{O} / \mathrm{HDO}, \mathrm{H}_{2} \mathrm{CO}$, $\mathrm{CH}_{3} \mathrm{OH}, \mathrm{CH}_{4}, \mathrm{CO}_{2}, \mathrm{~N}_{2}, \mathrm{~N}_{2} \mathrm{H}_{2}, \mathrm{HNO}, \mathrm{NH}_{3}, \mathrm{HCN}, \mathrm{OCN}$, and $\mathrm{H}_{2} \mathrm{CN}$. The total network includes 6284 reactions and 665 species. The initial chemical abundances input into our model are discussed in Appendix B (see Table 3).

We constrain the $\mathrm{CO}$ and nitrogen abundances using observations of $\mathrm{CO}$ and $\mathrm{HCN}$ (see Appendix B for more details). Regarding CO, motivated by the results of Favre et al. (2013) where CO is highly depleted in $\geq 20 \mathrm{~K}$ gas by $1-2$ orders of magnitude, we manually change (reduce) the initial CO abundance until our models reproduce the CO isotopologue observations after 1 Myr of chemical evolution. Chemical processes within the disk naturally remove $\mathrm{CO}$ from the gas over time by converting CO into more complex carbon-bearing ices (Bergin et al. 2014); however, we find that starting with a "normal" CO abundance of $\chi(\mathrm{CO})=10^{-4}$ and allowing the chemistry to evolve over 3-10 Myr does not alone sufficiently reduce the $\mathrm{CO}$ abundance to match the observed $\mathrm{C}^{18} \mathrm{O}$ and ${ }^{13} \mathrm{CO}$ observations. This finding implies that some type of additional CO depletion is necessary. Possible mechanisms include CO chemical conversion to organic ices at even earlier stages, prior to disk formation (i.e., the disk does not start out with $\chi(\mathrm{CO})=10^{-4}$ ) or, alternatively, freeze-out of CO-derived ices in the disk combined with rapid settling of large, ice-coated grains to the midplane, which can remove carbon and oxygen from the upper layers of the disk. With our present simple reduction models, we confirm the low gas-phase $\mathrm{CO}$ abundance posited in Favre et al. (2013), where we derive a $\mathrm{CO}$ abundance of $\chi(\mathrm{CO})=10^{-6}$ relative to $\mathrm{H}_{2}$ (traced by HD).

The chemical calculations are explicitly nonequilibrium, and as such, there is some uncertainty on what "chemical time" to adopt when comparing model results with observations. Time "zero" in the chemical code corresponds to a fixed physical structure with uniform input abundances set by Table 3 at every location in the disk. As time progresses there is a net gas-phase depletion because there are several "sinks" in the network, where energetic $\mathrm{He}^{+}$atoms break molecular bonds and gradually form more complex species with higher grain-surface binding energies than the parent molecule (for the case of CO, see Bergin et al. 2014, and \$.1.

The time for this sequestration process is related to the freeze-out time, which is directly proportional to the typical size of grains assuming a constant gas-to-dust mass ratio (e.g., Aikawa et al. 1996). Specifically, larger grains have less surface area per unit volume of gas than 
Table 1

Spectrally integrated TW Hya line fluxes used to constrain the physical model. Reported uncertainties on the molecular ions include statistical errors and a $15 \%$ systematic uncertainty in the absolute flux scale.

\begin{tabular}{|c|c|c|c|c|}
\hline Line & $\begin{array}{l}\text { Integrated Line Flux } \\
(\mathrm{Jy} \mathrm{km} / \mathrm{s})\end{array}$ & $\begin{array}{c}\text { Beam } \\
(\text { maj } \times \text { min, PA })\end{array}$ & Reference & Recover all? \\
\hline $\mathrm{HCO}^{+}(1-0)$ & $0.85 \pm 0.14$ & $\left(4.2^{\prime \prime} \times 2.9^{\prime \prime}, 72^{\circ}\right)$ & ALMA 2011.0.00001.SV. & $\mathrm{Y}$ \\
\hline $\mathrm{HCO}^{+}(3-2)$ & $12.9 \pm 2.12$ & $\left(0.69^{\prime \prime} \times 0.39^{\prime \prime}, 16.9^{\circ}\right)$ & This work. & Y \\
\hline $\mathrm{HCO}^{+}(4-3)$ & $23.3 \pm 3.5$ & $\left(1.7^{\prime \prime} \times 1.6^{\prime \prime}, 18^{\circ}\right)$ & ALMA 2011.0.00001.SV. & $\mathrm{N}$ \\
\hline $\mathrm{H}^{13} \mathrm{CO}^{+}(3-2)$ & $0.7 \pm 0.18$ & $\left(1.81^{\prime \prime} \times 0.90^{\prime \prime}, 15.6^{\circ}\right)$ & This work & $\mathrm{Y}$ \\
\hline $\mathrm{H}^{13} \mathrm{CO}^{+}(4-3)$ & $1.1 \pm 0.41$ & $\left(4.1^{\prime \prime} \times 1.8^{\prime \prime}, 3.3^{\circ}\right)$ & Qi et al. (2008) & $\mathrm{N}$ \\
\hline $\mathrm{N}_{2} \mathrm{H}^{+}(3-2)$ & $2.2 \pm 0.46$ & $\left(3.5^{\prime \prime} \times 2.0^{\prime \prime}, 10^{\circ}\right)$ & Qi et al. (2013a) & $\mathrm{Y}$ \\
\hline $\mathrm{N}_{2} \mathrm{H}^{+}(4-3)$ & $4.6 \pm 0.7$ & $\left(0.63^{\prime \prime} \times 0.59^{\prime \prime},-18^{\circ}\right)$ & Qi et al. (2013b) & $\mathrm{N}$ \\
\hline HD $(1-0)$ & $70.6 \pm 7.8$ & $\left(9.4^{\prime \prime} \times 9.4^{\prime \prime}\right)$ & Bergin et al. (2013) & $\mathrm{Y}$ \\
\hline $\mathrm{C}^{18} \mathrm{O}(2-1)$ & $0.68 \pm 0.18$ & $\left(2.8^{\prime \prime} \times 1.9^{\prime \prime},-1.3^{\circ}\right)$ & Favre et al. (2013); Qi et al. (2013b) & Y \\
\hline${ }^{13} \mathrm{CO}(2-1)$ & $2.76 \pm 0.18$ & $\left(2.7^{\prime \prime} \times 1.8^{\prime \prime},-3^{\circ}\right)$ & Favre et al. $(\overline{2013}) ;$ Qi et al. (2013b) & $\mathrm{Y}$ \\
\hline $\mathrm{HCN}(3-2)$ & $8.5 \pm 1.7$ & $\left(1.6^{\prime \prime} \times 1.1^{\prime \prime},-0.5^{\circ}\right)$ & Q1 et al. $(2008)$ & $\mathrm{Y}$ \\
\hline
\end{tabular}
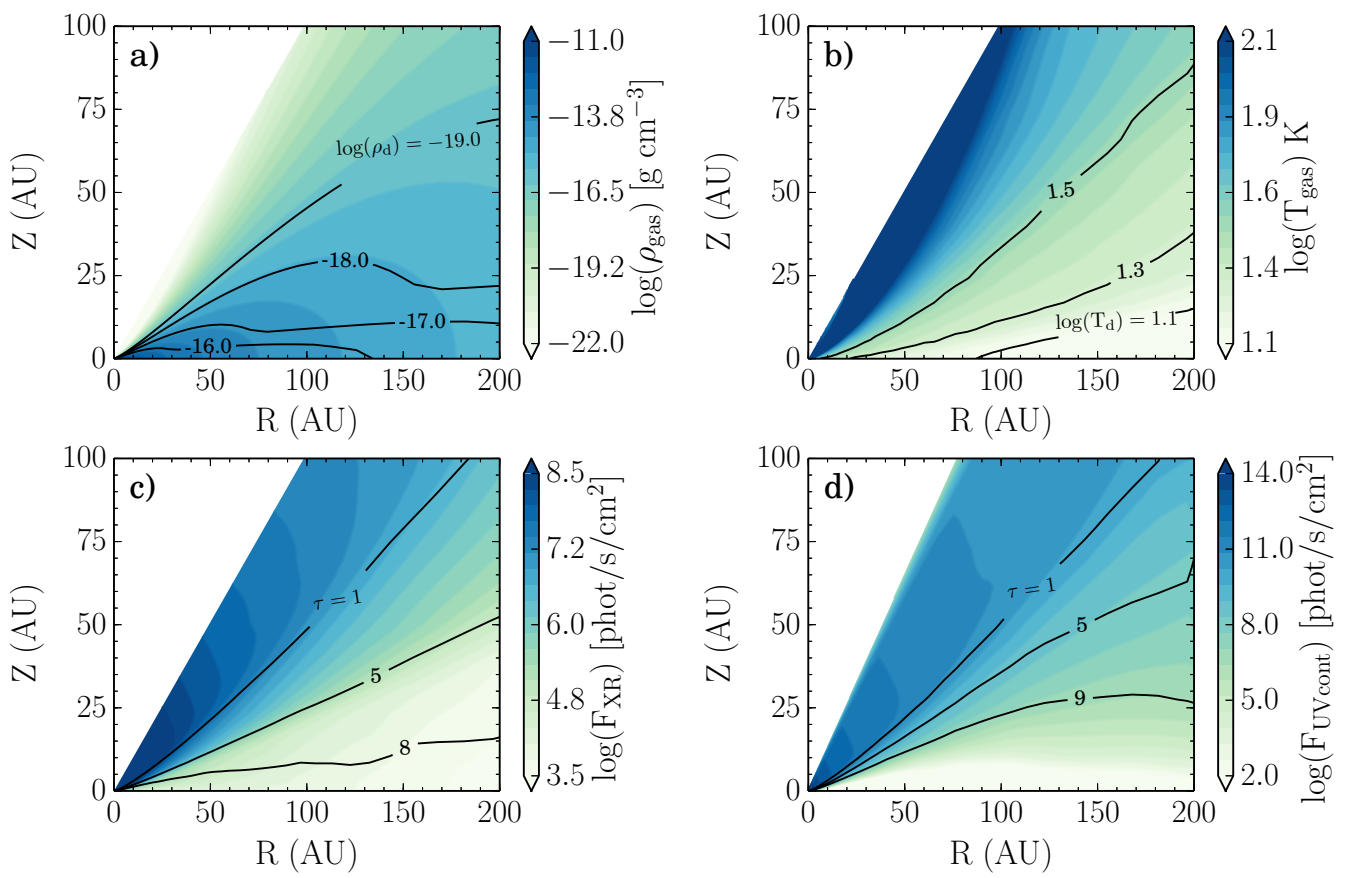

Figure 3. TW Hya physical model constrained from the SED and HD observations (see Appendix C). The filled contours in panels a) and b) are the gas density and temperature, respectively. The overlaid contour lines in a) and b) show the corresponding dust density and temperature. Panel c) plots the integrated X-ray flux between 1-20 keV with lines of wavelength integrated optical depth over plotted. Similarly, panel d) shows the wavelength integrated UV flux along with corresponding UV optical depth.

smaller grains, i.e., the total surface area per unit volume is $\sigma_{g}(r) n_{g}\left(r_{g}\right) \propto r_{g}^{2} r_{g}^{-3.5}=r_{g}^{-1.5}$, where $n_{g}$ is the volume number density of grains and $\sigma_{g}$ is the surface area of a single grain. Therefore, as grains grow larger, the surface area of grains drops and molecule collisions with grains are less frequent, slowing down the chemical time for freeze-out. Likewise, settling removes some fraction of the dust mass from the upper layers and correspondingly, dust surface area, also slowing down the rate of subsequent freeze-out. Grain growth and settling therefore slow the chemistry's natural tendency to sequester volatiles into more complex ices, and an old disk with large grains can look like a young disk with small grains. Thus grain growth can reduce the "chemical age" as compared to the physical (stellar) age.

In the present model, owing to the settled nature of the disk, we reduce the "chemically active" surface area of grains to $3 \%$ of its standard value, i.e., compared to a uniform gas-to-dust mixture (mass ratio of 100) and grains typically $r_{q}=0.1 \mu \mathrm{m}$ in size, the default assumption of the Fogel et al. (2011) model. Because the typical grain surface area is a single parameter in the model, we cannot increase the surface area in the midplane where the larger $(\geq 1 \mathrm{~mm})$ grains will collect. However, these larger grains contribute negligibly to the surface area compared to the small grains, which are also present throughout the midplane (e.g., Dullemond \& Dominik 2004). This change in grain surface area increases the time-scale for freeze-out by a factor of $\sim 30$.

Taking these factors into consideration and the unknown age of the TW Hya star and disk system of $\sim 3-10$ Myr (Barrado Y Navascués 2006 Vacca \& Sandell 2011), we opt to examine the time-evolved chemical abundances at $1 \mathrm{Myr}$, when the ion-chemistry has 
Table 2

Incident $\mathrm{CR}$ model ionization rates, $\zeta_{\mathrm{CR}}$.

\begin{tabular}{lcr}
\hline \hline Model & ID & \multicolumn{1}{c}{$\zeta_{\mathrm{CR}}$} \\
\hline Moskalenko et al. $(2002)$ & M02 & $6.8 \times 10^{-16} \mathrm{~s}^{-1}$ \\
Webber (1998) & W98 & $2 \times 10^{-17} \mathrm{~s}^{-1}$ \\
Wolar System Minimum & SSM & $1.1 \times 10^{-18} \mathrm{~s}^{-1}$ \\
Solar System Maximum & SSX & $1.6 \times 10^{-19} \mathrm{~s}^{-1}$ \\
T Tauri Minimum & TTM & $7.0 \times 10^{-21} \mathrm{~s}^{-1}$ \\
\hline
\end{tabular}

leveled to an approximate steady-state. Because we cannot take into account the time-evolving grain size and its changing spatial distribution simultaneously with the time evolution of the chemistry along with uncertainties in stellar ages, calibrating the chemical evolution using molecular line observations is a better approach to assessing the current evolutionary state of the TW Hya molecular gas disk. In this light, the present model is verified as appropriate to the current chemical evolutionary stage of TW Hya using neutral gas constraints, CO and HCN, as described in Appendix B.

\subsection{Ionization}

The primary sources of ionization considered in this work are X-rays, CRs, and UV photoionization. X-rays and CRs are the most important for the ionization of the dense molecular gas, where both are capable of ionizing $\mathrm{H}_{2}$ and helium. Both the incident CR flux and the implementation of X-rays (motivated by X-ray observations of the source) are the primary free parameters of our modeling efforts and are described in detail below. We do not include the ionization contribution from the decay of SLRs, owing to the advanced age of TW Hya (3-12 Myr; Webb et al. 1999, Barrado Y Navascués 2006, Mentuch et al. 2008; Vacca \& Sandell 2011; Weinberger et al. 2013) and its relative isolation from recent massive star formation, though see $\$ 4.3$ for additional discussion.

\subsubsection{CR Ionization}

The $\mathrm{CR}$ ionization rate, $\zeta_{\mathrm{CR}}$, is a free parameter in the present study, where we have adopted the results of Cleeves et al. (2013a) to construct five input cosmic ray models. These models consist of empirically motivated CR particle spectra and include self-consistent energy decay with depth (i.e., surface density). The CR attenuation is taken in the vertical direction for ease of calculation in the chemical models. In Table 2 we provide the incident $\mathrm{CR}$ ionization rate at the surface of the disk (prior to attenuation by the gas itself). Details regarding the calculation of $\zeta_{\mathrm{CR}}$ and the functional form of the decay with surface density can be found in Cleeves et al. (2013a). In summary, the Moskalenko et al. (2002) (M02) ionization rate is similar to that determined for the diffuse ISM, the Webber (1998) (W98) rate is consistent with values for the dense, molecular ISM, and Solar System Minimum (SSM) and Solar System Maximum (SSX) are the present day CR fluxes at 1 AU. T Tauri Minimum is an "extreme" modulation case, extrapolated from the solar values (see Cleeves et al. 2013a, for details).

\subsubsection{X-ray Ionization}

The efficiency of X-rays to ionize a disk depends both on the total flux and the hardness of the spectra. TW
Hya has a substantial measured X-ray luminosity of $L_{\mathrm{XR}} \sim 2 \times 10^{30} \mathrm{erg} \mathrm{cm}^{-2} \mathrm{~s}^{-1}$ (e.g., Kastner et al. 1999 , Raassen 2009, Brickhouse et al. 2010), assuming a distance of $d=55$ pc. This flux has been observed to double or triple during X-ray flares over periods of hours (Kastner et al. 2002 Brickhouse et al. 2010) at a cadence of less than a day (Kastner et al. 1999).

The quiescent state X-ray spectrum is well-fit by a two temperature plasma model with characteristic temperatures of 2-3 MK and 10-12 MK (Kastner et al. 1999. Brickhouse et al. 2010), which are associated with X-ray emission from the accretion shock (Calvet \& Gullbring 1998; Kastner et al. 1999, 2002, Stelzer \& Schmitt|2004] Brickhouse et al. 2010; Dupree et al. 2012), and coronal emission from hot plasma (e.g., Lamzin 1999, Güdel \& Telleschi 2007; Brickhouse et al. 2010), respectively. During flares, hints of a hard X-ray excess have been observed by Kastner et al. (1999). Kastner et al. (2002) and Brickhouse et al. (2010) also found that during independent flaring events, harder energy bands and high temperature diagnostics were affected by the flare while the soft component was not.

Despite the exquisite data available on X-ray fluxes and spectra toward TW Hya, its impact on ionization and chemistry is highly uncertain. First, the shape of the spectrum beyond $E_{\mathrm{XR}} \sim 3 \mathrm{keV}$ (the very photons that ionize the bulk gas) in both the quiescent and flaring states is not directly known. Second, the variability of the X-ray flux and possibly spectrum occurs on small enough time scales that the chemistry may not be able to reset between flares, and may thus reflect the flared state of TW Hya dependent on the relevant timescales. For ion chemistry the relevant time scale is the electron recombination rate. The typical electron recombination rate coefficient is of order $\alpha_{\text {rec }} \sim 1 \times 10^{-7} \mathrm{~cm}^{3} \mathrm{~s}^{-1}$. The electron density at the inner disk surface, $R=30 \mathrm{AU}$, $Z=12$ AU (i.e., above the $\tau_{\mathrm{XR}} \sim 1$; Figure 3), is approximately $n_{e} \sim 10 \mathrm{~cm}^{-3}$, resulting in a recombination time of $t_{\text {rec }} \sim\left(\alpha_{\text {rec }} n_{e}\right)^{-1}=12$ days. Therefore, if the flaring time scale is on the order of days, the chemistry may not have time to "reset" between flares.

To address this issue, we treat the X-ray flux and spectrum as a free parameter in our model. We construct four low-resolution X-ray templates with XSPEC6 where the baseline "quiescent" X-ray model is the two component plasma model (raymond) derived by Kastner et al. (1999), with $1.7 \mathrm{MK}$ and 9.7 MK components (these components are similar to those found in the detailed spectroscopic study of Brickhouse et al. $(2010))$. The luminosity in the quiescent template is $L_{\mathrm{XR}}=1.5 \times 10^{30} \mathrm{erg} \mathrm{cm}^{-2} \mathrm{~s}^{-1}$. To approximate the X-ray flaring state, on top of the quiescent spectrum we add an artificial hard component at $4 \mathrm{keV}$, changing the relative amounts of hard-to-soft X-rays and thus decreasing the overall spectral slope (Figure 4).

We fix the soft X-ray flux at $1 \mathrm{keV}$ across the four spectra based upon the results that the low temperature, softer X-ray component is unaffected by the observed flares. Between the four spectra considered here, the integrated X-ray luminosity changes by a factor of just $\sim 3$ (see Figure 4 legend); however, the specific flux at

\footnotetext{
${ }^{6}$ http://heasarc.gsfc.nasa.gov/xanadu/xspec/.
} 


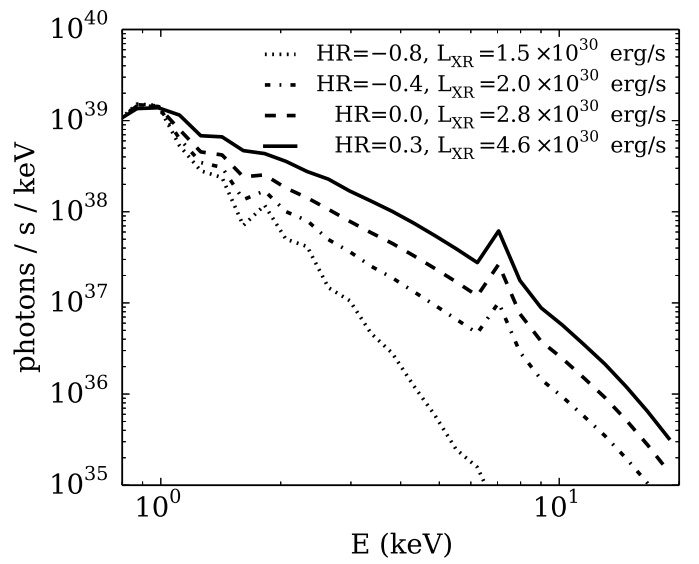

Figure 4. Template X-ray spectra for the ionization model grid, where the softest X-ray spectrum (i.e., the quiescent template), $\mathrm{HR}=-0.8$, is motivated by the observations of Kastner et al. (1999 2002), while the other spectra are artificially hardened to simulate different degrees of "flaring" spectral states.

the hard X-ray tail ( $\left.E_{\mathrm{XR}} \gtrsim 5 \mathrm{keV}\right)$ changes by an order of magnitude. All but the highest energy flaring spectrum are consistent within the error bars of the data presented in Kastner et al. (1999). We identify the four models by their X-ray hardness ratio, which is defined as the ratio $\left(L_{\text {soft }}-L_{\text {hard }}\right) /\left(L_{\text {soft }}+L_{\text {hard }}\right)$ where $L_{\text {soft }}$ is the X-ray luminosity between $0.5-2.0 \mathrm{keV}$ in $\mathrm{erg} \mathrm{s}^{-1}$ and $L_{\text {hard }}$ is the equivalent between $2.0-10 \mathrm{keV}$. More negative numbers are soft X-ray dominated, while more positive numbers are hard X-ray dominated.

We calculate the $\mathrm{X}$-ray propagation throughout the disk for energies of $E_{\mathrm{XR}}=1-20 \mathrm{keV}$ in $1 \mathrm{keV}$ intervals as described in (Cleeves et al. 2013a) with the Monte Carlo code presented in Bethell \& Bergin (2011b). The absorption cross-sections are provided in Bethell \& Bergin (2011a) and the X-ray scattering is dominated by Thompson scattering. Next, we apply the template spectra in Figure 4 to determine the X-ray radiation field as a function of energy and position within the disk, which is the input for the chemical calculations. The X-rays primarily ionize $\mathrm{H}_{2}$ and $\mathrm{He}$, where we adopt the ionization cross-sections provided in Yan et al. (1998), integrated over the local X-ray spectrum. The same Bethell \& Bergin (2011b) code is also used for the UV calculations (see Cleeves et al. 2014a, for our treatment of the Lyman- $\alpha$ transfer and A.2). The energy-integrated Xray and UV field $(930-2000 \AA)$ are shown in Figure 3 c and $3 \mathrm{~d}$, respectively, along with their total optical depth.

\subsection{Line Radiative Transfer and Synthetic Observations}

The set of five CR models (\$3.3.1) and four X-ray models (\$3.3.2) forms a grid of twenty different ionization profiles for a fixed physical (density and temperature) structure (Appendix A). For each model, we calculate the time-dependent chemistry as described in $\$ 3.2$ To enable model-data comparison, we then calculate the emergent line emission from the abundances and model physical conditions assuming a distance of $d=55 \mathrm{pc}$ and, for cases where spatial filtering cannot be excluded, we also simulate the particular observations.

The line radiative transfer is computed using the LIME code (Brinch \& Hogerheijde 2010) in non-LTE where

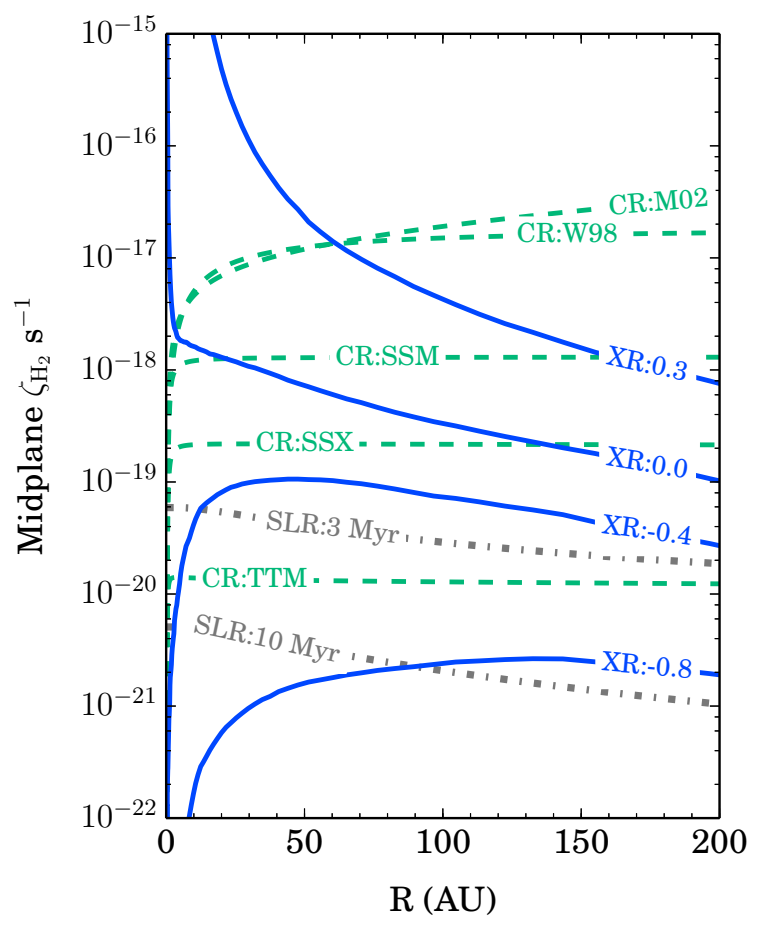

Figure 5. $\mathrm{H}_{2}$ ionization rate in the midplane $(z=0 \mathrm{AU})$ due to $\mathrm{X}$-rays $(\mathrm{XR})$, cosmic rays $(\mathrm{CR})$ and short-lived radionuclides (SLR). Incident (unattenuated) CR fluxes are listed in Table 2 $\mathrm{X}$-rays are labeled by their hardness ratio (see also Figure 4 for the incident spectra). SLR rates are determined from the initial solar nebular abundances with the indicated decay time (and no late stage injection).

available 7 . We consider two components to the gas velocity: i) Keplerian rotation around the star based on the stellar mass and viewing geometry $\left(i \sim 7^{\circ}\right)$ and ii) the gas turbulent velocity, i.e., the doppler-B parameter. We adopt a turbulent velocity of $40 \mathrm{~m} \mathrm{~s}^{-1}$ based on the observed upper limit from Hughes et al. (2011). The end product of the LIME simulations are data cubes, i.e., two dimensions on the plane of the sky and a third in velocity. Since carbon and oxygen isotopologues are not considered independently in the chemical network, their abundances are calculated based on the main isotopologues using a fixed ratio of ${ }^{12} \mathrm{C} /{ }^{13} \mathrm{C}=70 \pm 10$ and ${ }^{18} \mathrm{O} /{ }^{16} \mathrm{O}=540 \pm 30$ (Henkel et al. 1994 Prantzos et al. 1996) appropriate for the local ISM. For all lines, we simulate the line and continuum emission simultaneously with the dust and gas co-spatial within the framework of the physical model. We then use self-consistent dust opacities from the radiative transfer and UV modeling, and then subtract off the continuum emission when comparing the line fluxes.

The calculated line emission fluxes are compared to observations to determine the goodness of fit of the chemical simulations. This can be done directly using the LIME output for lines observed for the observations that have sufficient short spacings to not have any flux resolved out, marked as 'Recover all?'='yes' in Table 1. Exceptions to this are the $(4-3)$ rotational lines of

${ }^{7}$ Collisional rates for $\mathrm{HCO}^{+}, \mathrm{H}^{13} \mathrm{CO}^{+}$and $\mathrm{N}_{2} \mathrm{H}^{+}$are compiled at the Leiden LAMDA database (Schöier et al. 2005), where the primary literature for the spectroscopic data is Anderson et al. (1977); Sastry et al. (1981); Botschwina et al. (1993); Flower (1999). 
$\mathrm{HCO}^{+}, \mathrm{H}^{13} \mathrm{CO}^{+}$, and $\mathrm{N}_{2} \mathrm{H}^{+}$. For these lines, we make synthetic observations using the simobserve task in $\mathrm{CASA}^{8}$ and the specific array configurations used for these SMA and ALMA observations. The $\mathrm{HCO}^{+}(4-3)$ and $\mathrm{N}_{2} \mathrm{H}^{+}(4-3)$ were both observed as part of ALMA Early Science operations, where the former was a Science Verification target and the latter was reported in Qi et al. (2013b). Because the array configurations during these observations were non-standard, we used the original data to create the observation specific array configurations for the simobserve task. From the simulated visibilities we reconstruct the on-sky image using clean with natural weighting. The simulated beam from the reconstructed images is $\left(4.1^{\prime \prime} \times 1.97^{\prime \prime}\right)$ and $\left(0.66^{\prime \prime} \times 0.56^{\prime \prime}\right)$ for $\mathrm{HCO}^{+}(4-3)$ and $\mathrm{N}_{2} \mathrm{H}^{+}(4-3)$, respectively, which are in good agreement with the beams from the observations (see Table 1). Likewise, the CASA simulations for the SMA H ${ }^{13} \mathrm{CO}^{+}(4-3)$ with a beam of $\left(4.1^{\prime \prime} \times 1.97^{\prime \prime}\right)$ reasonably agrees with the observed beam.

For all lines except $\mathrm{HCO}^{+}(3-2)$ and $\mathrm{N}_{2} \mathrm{H}^{+}(4-3)$, we only compare spatially/spectrally integrated line fluxes. To determine quality of fit, we combine the uncertainties on the original observations in quadrature with a $10 \%$ uncertainty from the stochastic point-casting uncertainty of the LIME code (Brinch \& Hogerheijde 2010). For the uncertainty of the two $\mathrm{H}^{13} \mathrm{CO}^{+}$rotational lines, we also include a $14.3 \%$ uncertainty for the isotopologue ratio of ${ }^{12} \mathrm{C} /{ }^{13} \mathrm{C}=70 \pm 10$.

For the best spatially resolved data, $\mathrm{HCO}^{+}(3-2)$ and $\mathrm{N}_{2} \mathrm{H}^{+}(4-3)$, we directly compare the observed emission profile on the plane of the sky to the model emission profile. To assess the fit to the model emission profiles, we measure the difference between the model and observed line fluxes in units of the integrated $\sigma$ $\left(\mathrm{Jy} / \mathrm{beam} \mathrm{km} \mathrm{s}^{-1}\right.$ ) over the angular extent of the disk. From this difference, we determine the radial difference in units of the uncertainty on the line flux, $\sigma(R)$. From this profile, we determine a disk-averaged $\sigma$ between the model and observation by integrating over the disk area, $\int 2 \pi \Delta \sigma(\theta) \theta d \theta / \int 2 \pi \theta d \theta$, which implicitly weights towards the outer disk because it covers more of the total emission area. For both $\mathrm{HCO}^{+}(3-2)$ and $\mathrm{N}_{2} \mathrm{H}^{+}(4-3)$ we integrate out to $\Delta \theta=3^{\prime \prime}$ from the stellar position, beyond which the emission drops off substantially.

\section{RESULTS}

\section{1. $\mathrm{HCO}^{+}$Spatial Distribution}

The $\mathrm{HCO}^{+}(3-2)$ velocity integrated line emission for the combined data set and the VEX data set are shown in Figure 1 together with the simultaneously observed continuum data. The VEX data clearly deviates from axisymmetry, and while less pronounced, this deviation is also recovered in the combined image. The VEX emission excess is separated from the phase center by the size of the $\sim 25$ AU beam and seems to be unresolved. In the combined image, the feature shows up as a $3 \sigma$ enhancement as compared to the emission profile across the rest of the disk at the same radial distance. The emission contributes approximately $\sim 15-20 \%$ of the overall disk-integrated $\mathrm{HCO}^{+}(3-2)$ flux, which is a substantial amount of the total emission confined to a

${ }^{8}$ http://casa.nrao.edu/ very small region. The possible origin of this asymmetry is discussed in 55.3 . While the asymmetry is not the focus of the present paper, equipped with this additional spatial information, we are able to exclude the southwest quadrant from the ionization fitting as it does not reflect the majority of the disk properties. The three other quadrants are assumed to be representative of the axisymmetric $\mathrm{HCO}^{+}(3-2)$ emission profile. The remaining $\mathrm{HCO}^{+}$lines, $(1-0),(4-3)$ and the isotopologues, were observed at lower spatial resolution (see Table 1 . and as a result, we are unable to correct their emission for such an asymmetry if it is a long-lived feature. These observations highlight the utility of high spatial resolution observations even for the study of bulk gas chemical properties.

\subsection{Model Grid Results}

The contribution of the different X-ray and cosmic ray models to the disk midplane ionization level is shown in Figure 5. We also plot the contribution from two SLR realizations assuming an age of $3 \mathrm{Myr}$ and $10 \mathrm{Myr}$ with Solar System-like initial SLR abundances (see Cleeves et al. 2013b for details). The relative importance of CR and $\mathrm{X}$-rays for $\mathrm{H}_{2}$ ionization clearly depends both on the $\mathrm{CR}$ attenuation and the X-ray hardness. In its quiescent state, even the most attenuated cosmic rays dominate the X-ray ionization level throughout the disk. Moderate hardening/flaring of the X-ray spectra results in an $\mathrm{X}$-ray dominated ionization profile in the inner disk, and for the hardest X-ray spectra, X-rays drive ionization throughout the disk for all cases with any CR attenuation. SLRs only contribute to the ionization level if the CRs are extremely attenuated and the star displays no X-ray flaring activity, justifying their exclusion from the model calculations.

Figure 6] shows the chemistry model results in terms of $\mathrm{HCO}^{+}$and $\mathrm{N}_{2} \mathrm{H}^{+}$radial column density profiles for the full grid of models i.e. the grid of $4 \times 5 \mathrm{X}$-ray and cosmic ray flux levels. Increasing X-ray hardness generally increases the $\mathrm{HCO}^{+}$and $\mathrm{N}_{2} \mathrm{H}^{+}$column densities. $\mathrm{CR}$ ionization does not strongly affect the $\mathrm{HCO}^{+}$column profile but does change the shape of $\mathrm{N}_{2} \mathrm{H}^{+}$, going from more centrally peaked column densities to radially flatter with increasing CR flux, similar to what was seen in the parameter study in Cleeves et al. (2014a) for a generic T Tauri disk model.

\subsection{Constraints on Ionizing Agents}

From the chemical models discussed in the previous section and the emission line analysis detailed in $\$ 4.2$ we can now compare the observations in Table 1 to the simulated molecular emission. The agreement between models and observed data is evaluated based on disk integrated fluxes for all lines except $\mathrm{HCO}^{+}(3-2)$ and $\mathrm{N}_{2} \mathrm{H}^{+}$ $(4-3)$, where the latter are fit based upon their radial emission profile (3.4). The results of this comparison are presented in Figure 7. For each considered emission line, agreement is classified as within $1 \sigma, 2 \sigma, 3 \sigma$ or as a poor fit between the observation and model. We also classify the overall quality of fit to each model in terms of 1) midplane ionization as probed by $\mathrm{N}_{2} \mathrm{H}^{+}$lines and 2) surface ionization tracers, i.e. $\mathrm{HCO}^{+}$lines. Finally, the total quality of fit is determined based on the fit to 

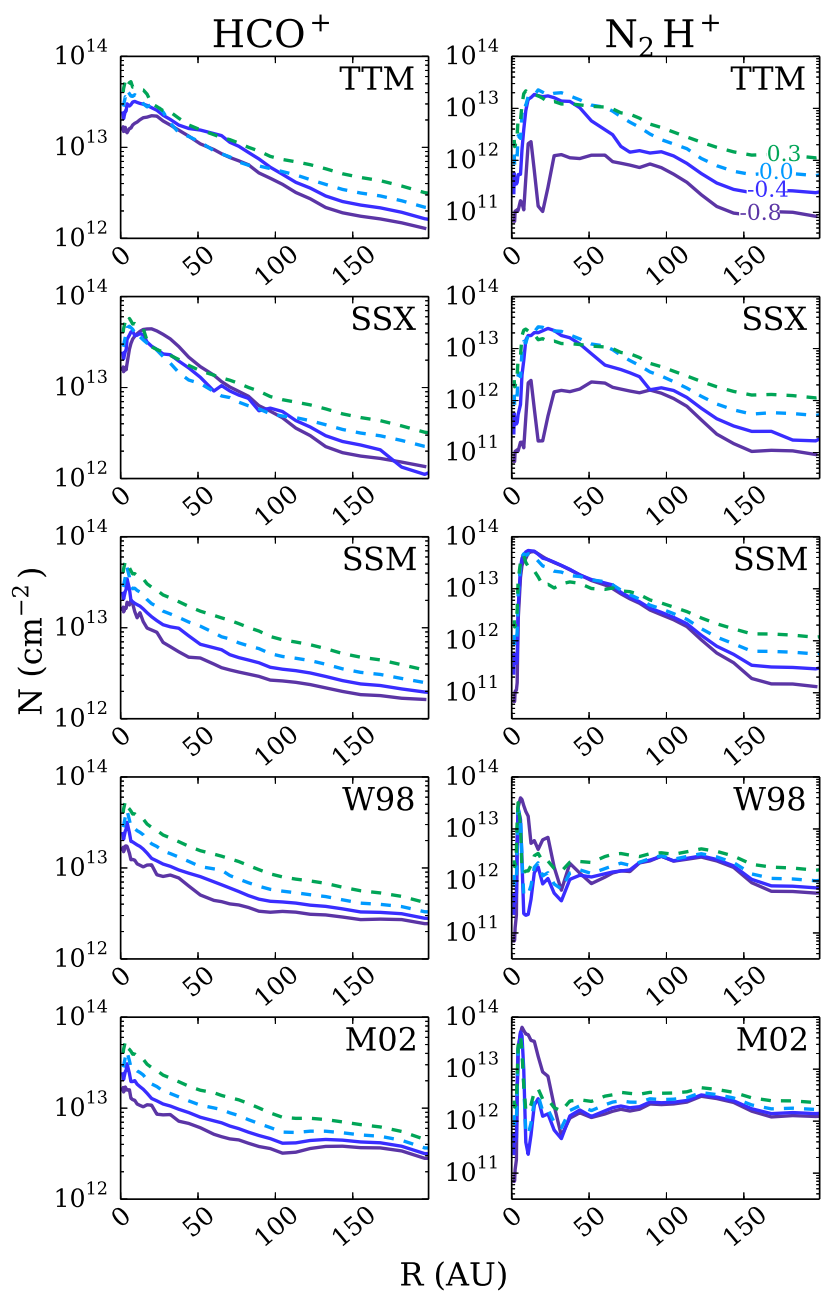

Figure 6. Vertically integrated column densities of $\mathrm{HCO}^{+}$(left) and $\mathrm{N}_{2} \mathrm{H}^{+}$(right) versus disk radius. Individual panels show increasing $\mathrm{CR}$ ionization rates from top to bottom. In each panel, variations due to changes in the $\mathrm{X}$-ray spectral hardness are shown by the different lines as labeled in the top right panel. The dashed lines indicate hard X-ray spectra, 0.0 and 0.3 , while the solid lines are the softer X-ray spectra, -0.8 and -0.4 .

midplane and surface ionization levels weighted equally, which implies that individual $\mathrm{N}_{2} \mathrm{H}^{+}$lines are weighted more heavily than individual $\mathrm{HCO}^{+}$lines for the final metric. Based upon these numbers, the best fit and acceptable fit models to both surface and midplane tracers are boxed in Figure 7.

Interestingly, all of the acceptable models have subinterstellar $C R$ ionization rates, $\zeta_{\mathrm{CR}} \lesssim 10^{-18} \mathrm{~s}^{-1}$. The best fit models, SSX and TTM, have $\zeta_{\mathrm{CR}} \lesssim 10^{-19} \mathrm{~s}^{-1}$. Additionally, these two models also have an X-ray spectral hardness ratio of -0.4 , which is harder than TW Hya's quiescent X-ray state, -0.8 , from the modelled observations of Kastner et al. (1999). This result implies that the chemistry seems to reflect an elevated X-ray ionization state perhaps as a result of the well-characterized frequent flaring behavior of the star (Kastner et al. 2002, Brickhouse et al. 2010).

The quality of fit is especially apparent in the emission profile fitting of $\mathrm{HCO}^{+}(3-2)$ and $\mathrm{N}_{2} \mathrm{H}^{+}(4-3)$, shown in Figures 8 and 9 and described in $\$ 3.4$. To evaluate the model goodness of fit for the relevant wedges in
Figure 7, the $\mathrm{HCO}^{+}(3-2)$ emission line models are convolved with a $\left(0.69^{\prime \prime} \times 0.39^{\prime \prime}\right)$ Gaussian beam, while the $\mathrm{N}_{2} \mathrm{H}^{+}(4-3)$ profile is measured from the CASA simulations discussed above. Both the observations and models are averaged over deprojected annuli assuming an inclination of $i=7^{\circ}$. The $\mathrm{HCO}^{+}(3-2)$ profile excludes the southwest quadrant from the annular averaging, along with the inner $0.3^{\prime \prime}$, due to the significant asymmetry present there.

Most models reproduce the radial distribution of $\mathrm{HCO}^{+}(3-2)$, while the $\mathrm{N}_{2} \mathrm{H}^{+}(4-3)$ spatial structure is more discriminatory between models. Specifically, Figure 9 shows that the $\mathrm{N}_{2} \mathrm{H}^{+}(4-3)$ ring discovered by Qi et al. (2013b) is narrow and the emission drops off steeply beyond the peak at $\theta \sim 0.7^{\prime \prime}$. These observed features are only reproduced by low, modulated CR models and moderately hardened X-ray spectra. The broad $\mathrm{N}_{2} \mathrm{H}^{+}$ emission extending to large angular scales in the high $\mathrm{CR}$ case is due to non-thermal desorption of $\mathrm{N}_{2}$ ice coupled with slower outer disk freeze-out times in the presence of CR ionization of $\mathrm{H}_{2}$. This exclusion of high $\mathrm{CR}$ models is independent of the specifics of the assumed disk density and temperature profile (Appendix C). There is thus very good agreement between the best-fit model derived from line flux comparisons and the spatial profiles of individual lines.

It is apparent from Figure 7 that the best fit models reproduce the $\mathrm{N}_{2} \mathrm{H}^{+}$emission better than the $\mathrm{HCO}^{+}$emission on the whole. The $\mathrm{HCO}^{+}$lines other than $(3-2)$ are better fit by very hard X-ray spectra (HR: 0.3) or a high $\mathrm{CR}$ rate and $\mathrm{HR}$ of 0.0 or 0.3 , and the models generally under predict the $\mathrm{HCO}^{+}$emission for all lines except the $(3-2)$ transition. Some of this under prediction may be explained by the observed asymmetric excess in the $(3-2)$ image (Fig. 1), which we were able to remove from the $\mathrm{HCO}^{+}(3-2)$ flux before model-data comparison, but may contribute significantly to the other line fluxes. A second source of error is the uniform reduction of the CO abundance (see Appendix B.1). There could be substantial spatial structure in the gas-phase $\mathrm{CO}$ abundance distribution, which is the precursor for $\mathrm{HCO}^{+}$formation. A third source of error is probably the details of the temperature structure, since the optically thin $\mathrm{H}^{13} \mathrm{CO}^{+}$lines, which should trace the column well, are better reproduced by the model than the optically thick low-spatial resolution lines, which should trace the disk (surface) temperature. There is clearly a need for future detailed thermal structure modeling using more higher resolution $\mathrm{HCO}^{+}$and $\mathrm{CO}$ data that can be used to optimize the temperature and chemical/physical structure simultaneously.

\section{DISCUSSION}

\subsection{The Ionization Environment of TW Hya}

From the emission modeling, we find that the CR ionization rate is substantially suppressed at all disk radii, with especially strong limits on the outer disk. One possible explanation is exclusion by young stellar winds as an analogue to the Solar System's heliosphere, i.e., a TTauriosphere. As was shown in Cleeves et al. (2013a) as well as Svensmark (2006); Cohen et al. (2012), winds from young stars should be efficient CR excluders if they operate over a large enough region of the disk. Alterna- 


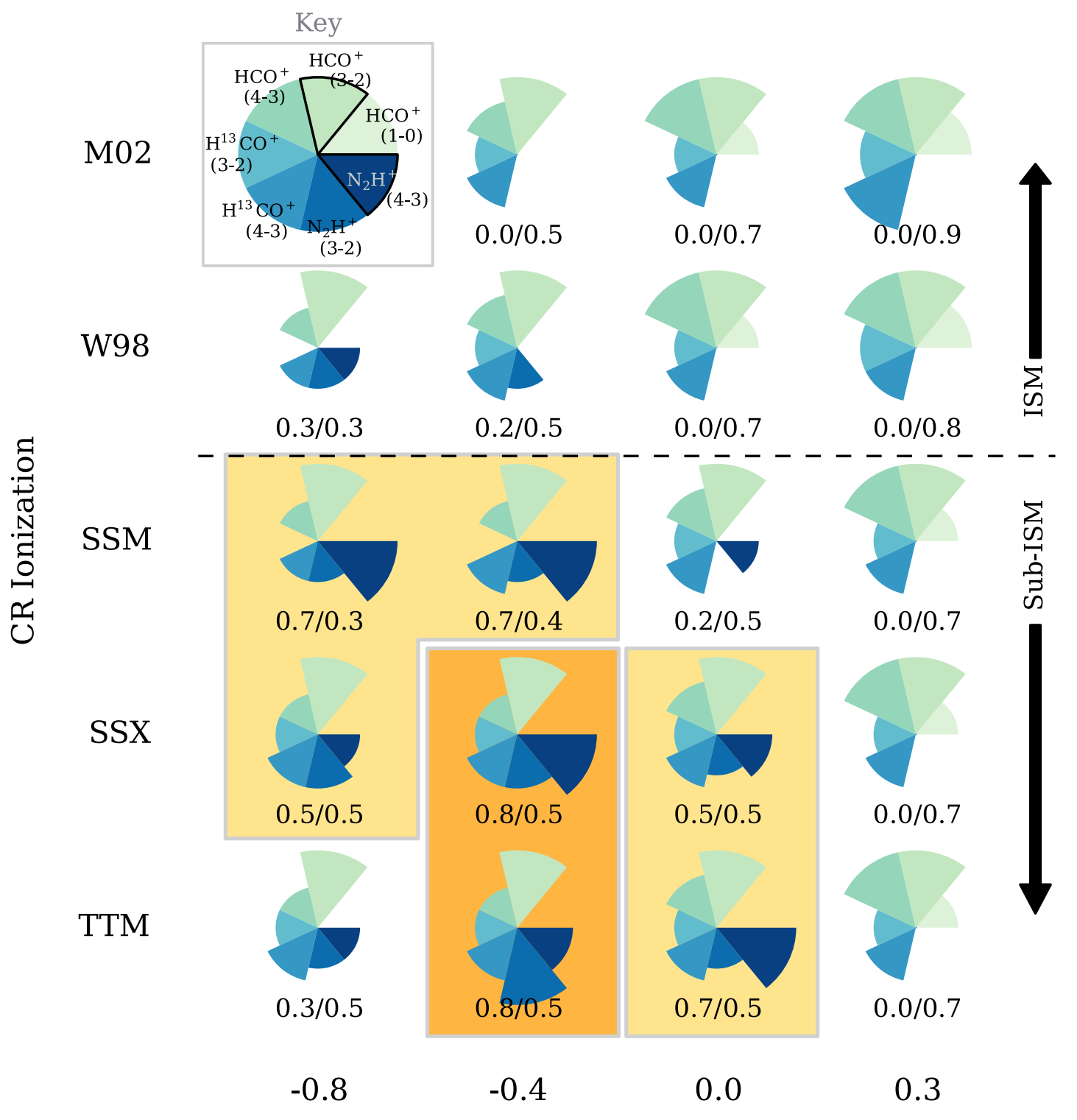

\section{Hard X-ray Ionization}

Figure 7. Ionization model goodness-of-fit for the lines indicated in the key (top-left). The size of the wedge indicates how closely the model matches the data, where the largest wedge matches within $1 \sigma$ (i.e., the best), the second and third smaller wedges indicate the model matches the data within $2 \sigma$ and $3 \sigma$ of the observations, respectively. No wedge implies the model and data do not match within $3 \sigma$. The wedges that are boxed in black in the key indicate lines that are fit by their emission profile rather than their integrated line flux. Columns are X-ray ionization increasing from left to right (see Fig. 4), while rows are CR ionization decreasing from the top down (see Table 2). The two numbers under each pie chart measure the goodness of fit to $\mathrm{HCO}^{+}$and $\mathrm{N}_{2} \mathrm{H}^{+}$line fluxes, respectively. We box in orange the best fit models, where the darkest orange corresponds to the best-fit model.

tively, if such winds are highly collimated by, e.g., magnetic fields, their covering fraction may be too small to shield the disk. Whether disk winds are also capable of excluding CRs should be explored in future work. If stellar or disk winds are the primary exclusion mechanism, they must operate well beyond the outer 200 AU gas disk radius, and the corresponding T-Tauriosphere, must be at least this large (see discussion in Cleeves et al. 2013a).

CRs can also be repelled by mirroring via external magnetic fields linking the disk to the parent cloud, especially if there are irregularities in the field lines (Padovani et al. 2013, Fatuzzo \& Adams 2014). Given TW Hya's relative isolation from molecular cloud material, it is uncertain whether such a large-scale environmental mag- netic field exists. Turbulent magnetic fields within the disk can also act as an additional source of energy loss for the CR particles if they are present (Dolginov \& Stepinski 1994); however, the disk must be sufficiently turbulent (and thus MRI-active) to support such a configuration.

The ionization rate derived for the midplane gas, $\zeta_{\mathrm{CR}} \lesssim 10^{-19} \mathrm{~s}^{-1}$, is, strictly speaking, a limit on all sources of ionization, including that due to SLRs and the scattered X-ray field in the midplane. If TW Hya had similar SLR abundances to the Solar Nebula and without significant additional late-stage SLR pollution by massive stars (Adams et al. 2014), the contribution from SLR ionization in TW Hya falls below the X-ray ionization rate in the midplane, or $\zeta_{\mathrm{XR}}=(2.3-10) \times 10^{-20} \mathrm{~s}^{-1}$, 
Low X-rays
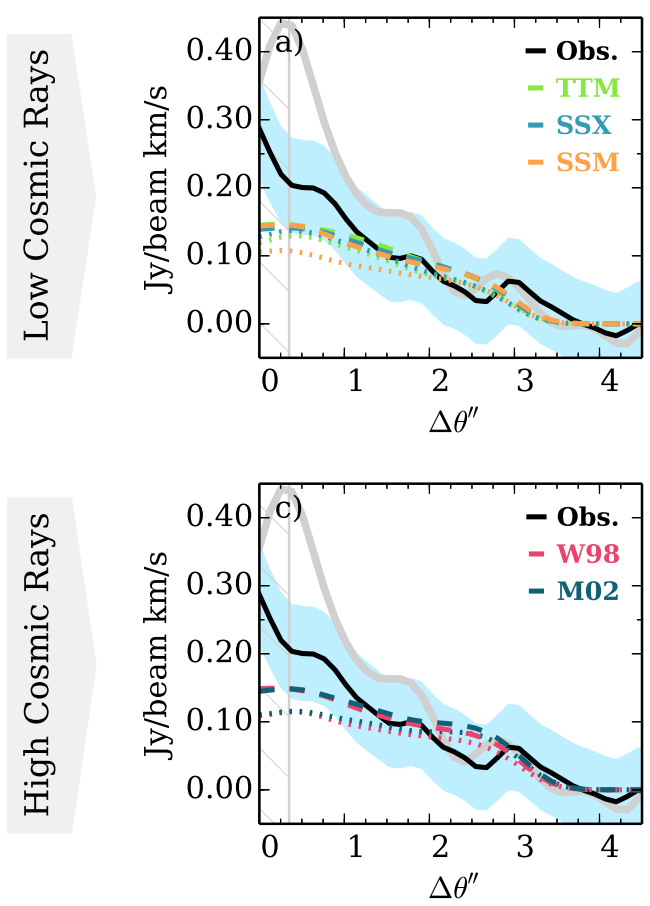

High X-rays
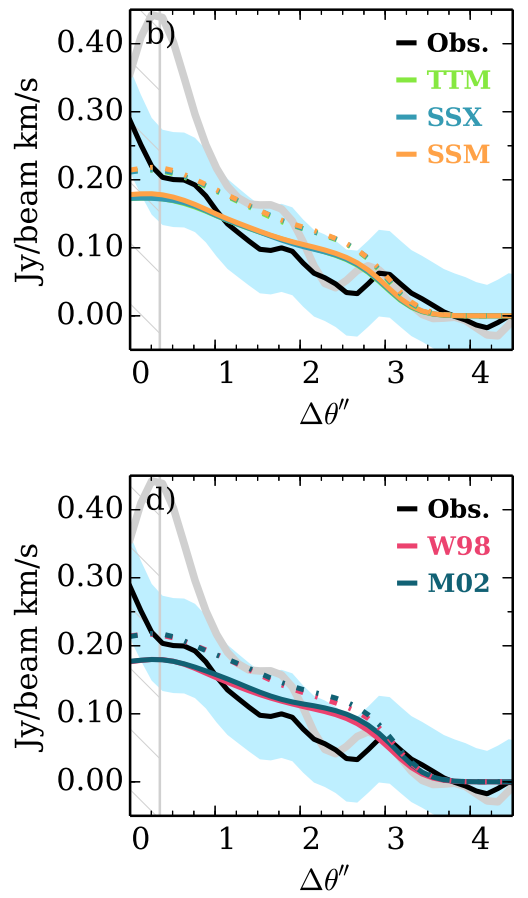

Figure 8. Integrated line intensity profile of $\mathrm{HCO}^{+}(3-2)$ versus angular distance from the star for the ionization models (colored lines) and the observed profile (solid black line). We have divided the models into low, modulated CR ionization rate models (top) and high, interstellar CR ionization rate models (bottom). The line style indicates the X-ray spectral hardness ratio (Fig. 4 where the two softer X-ray spectra are shown on the left panels: dotted $(-0.8)$ and dashed $(-0.4)$; and the two harder X-ray spectra are shown on the right: solid $(0.0)$ and dot-dashed (0.3). The inner vertical region hatched in gray in the $\mathrm{HCO}^{+}$profiles designates the part of the disk that is contaminated by the asymmetric feature (shown as the solid gray profile), which is excluded from the fitting.

at $\gtrsim 3$ Myr. For our best fit model, the scattered Xrays alone exceed the contribution from SLR ionization; thus further constraining the ionization rate due to SLR ionization will be difficult. Ionization tracers of the inner disk $(R<10 \mathrm{AU})$ midplane may help put more stringent limits on the dense gas ionization in the region where X-rays are highly attenuated and SLRs may dominate. Such small scales will be readily accessible by ALMA in the near future either with direct imaging or by using velocity information to spectrally resolve the inner disk.

The overall picture of TW Hya's relatively high surface ionization rate and ion-poor midplane is consistent with previous theoretical (e.g. Gammie 1996; Glassgold et al. 1997; Igea \& Glassgold 1999; Semenov et al.|2004; Semenov 2010; Semenov \& Wiebe 2011) and observational (Kastner et al. 1997; Qi et al. 2003; Dutrey et al. 2007 Öberg et al. 2011b) studies of disk ionization. With the IRAM Plateau de Bure Interferometry, Dutrey et al. (2007) conducted a search for $\mathrm{N}_{2} \mathrm{H}^{+}$and $\mathrm{HCO}^{+}$(and isotopologues) to measure the ionization state of three protoplanetary disks in a similar manner to the present paper. While the PdBI data had lower resolution and lower signal-to-noise, the $\mathrm{N}_{2} \mathrm{H}^{+}$observations of the three disks indicate a distinct drop at large radii, similar to what was found in the resolved TW Hya $\mathrm{N}_{2} \mathrm{H}^{+}(4-3)$ observations (see Fig. 9). Similar to our own findings (c.f. Cleeves et al. 2014a), the Dutrey et al. (2007) chemical models with CRs show a rise in $\mathrm{N}_{2} \mathrm{H}^{+}$column in the outer disk that is not present in the data (see their Figure 6 ). We attribute this rise in the models to outer disk CR ionization and decreased ion-recombination efficiency. While the lower signal-to-noise data presented in Dutrey et al. (2007) cannot definitively point to a CRpoor environment, these observations may hint that TW Hya's low CR environment is a common feature of protoplanetary disks.

\subsection{Dead-zones, Dust Growth and Accretion}

From our best fit ionization models we can estimate the size of the magneto-rotational instability (MRI) "dead" regions in the disk in a similar fashion to Cleeves et al. (2013a). In contrast to Cleeves et al. (2013a), we directly measure the ionization fraction from the chemical models, where the electron abundance is exactly equal to the ion abundance for a charge neutral disk. From the spatial ion abundances (equivalently the ion fraction), we estimate the magnetic Reynolds number, $R e$, and ambipolar diffusion coefficient, $A m$, to determine the approximate location of the MRI active versus dead layers. In Figure 10, we plot the electron abundance as filled contours.

We note that the electron abundance is slightly higher at the outer disk midplane in the present models than the steady-state estimate, $\chi_{e}=\sqrt{\zeta \alpha n\left(\mathrm{H}_{2}\right)}$, predicts because of the low densities and correspondingly longer recombination time-scales in the outer disk. We choose a critical $R e$ for the disk to be dead as $R e \leq 3300$ (where MRI active lies outside of the area inclosed by the thick purple line), but we also show $R e=1000$ and $R e=5000$ on the same plot, demonstrating that the $R e$-determined 

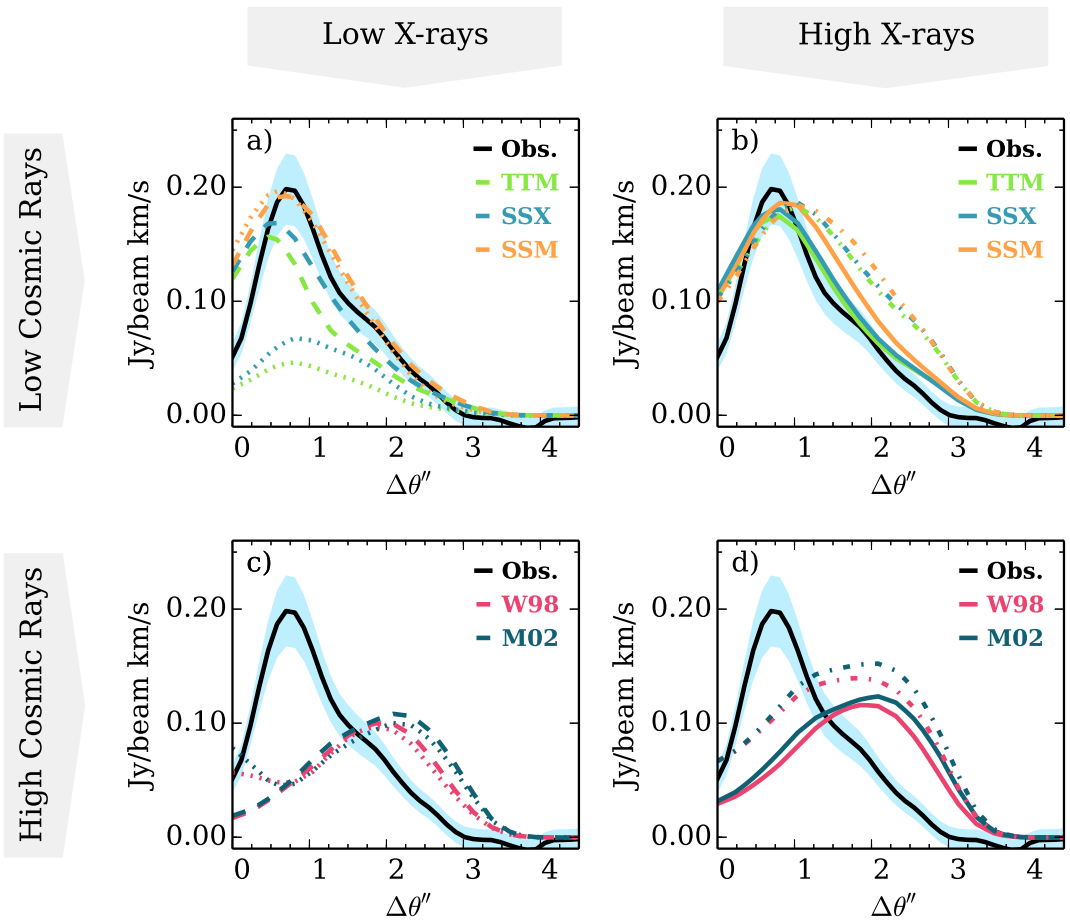

Figure 9. Integrated line intensity profile of $\mathrm{N}_{2} \mathrm{H}^{+}(4-3)$ versus angular distance from the star for the ionization models (colored lines) and the observed profile (solid black line). The panels are divided up as in Figure 8 The low CR models (top) are generally a better fit to the observed $\mathrm{N}_{2} \mathrm{H}^{+}(4-3)$ profile in tandem with a slightly harder X-ray profile, where the dashed line (top left panel, -0.4$)$ or the solid line (top right panel, 0.0) provide a good fit.

dead-zone is not very sensitive to this choice. Critical $A m<0.1$ (below which the disk is stable against the $\mathrm{MRI}$ ) is indicated by the region inside of the orange lines, and is mainly confined to a layer between the highly ionized surface and the outer disk midplane outside of $R>100$ AU (see grey contours of Fig. 10). Formally the disk must satisfy both $A m>0.1$ and $R e>3300$ to be active; however, the importance of the $A m$ criteria is affected by the strength and direction of the disk magnetic field, which is unconstrained here. Therefore we focus on $R e$ to approximately estimate the minimum region where the disk is dead versus active.

For the two best fit models, SSX and TTM (HR = -0.4 ), the $R e$ criteria results in a MRI dead region that extends from the inner disk out to $50 \mathrm{AU}$ and $65 \mathrm{AU}$ in the midplane (see Figure 10). This specific zone is of particular significance as it coincides with a region of concentrated large $(\mathrm{mm} / \mathrm{cm}$-sized) grains. Wilner et al. (2000) reported observations of TW Hya with the Very Large Array (VLA) where $7 \mathrm{~mm}$ continuum emission was found to be concentrated within a $R \sim 50$ AU radius along with an unresolved $3.6 \mathrm{~cm}$ detection within a $\sim 1.12^{\prime \prime} \times 0.93^{\prime \prime}(62 \times 51 \mathrm{AU})$ beam. Wilner et al. $(2005)$ reported $3.6 \mathrm{~cm}$ continuum observations at higher spatial $\left(0.15^{\prime \prime}\right)$ resolution and found that the emission was concentrated in a region of tens of AU in size. Deep $870 \mu \mathrm{m}$ observations with the Submillimeter Array (SMA) show similar morphology, where grains are concentrated within $R \sim 60$ AU of the parent star (Andrews et al. 2012), also seen in CARMA $1.3 \mathrm{~mm}$ observations where Isella et al. (2009) find a disk radius of $\sim 73 \mathrm{AU}$.

The co-spatial location of the dead-zone with the large grains is perhaps not coincidental. Gressel et al. (2012) argue that the decrease in turbulent activity within a dead-zone leads to the survival and growth embedded planetesimals, and thus may facilitate the growth of planets. Additionally Matsumura \& Pudritz (2005) and Matsumura et al. (2007) argue that the presence of deadzones may also halt rapid inward migration, leading to long-term survival of the planetesimal bodies. We note there are certainly other explanations for the dust concentration and the sharpness of the mm-grain outer edge, in particular as a natural outcome of the velocity dependence of radial drift (c.f., Birnstiel \& Andrews 2014); however, a change in the disk turbulent properties resulting from a dead-zone may facilitate further dust growth via sticking collisions within this region.

If such substantial dead-zone exists, it may also pose a problem for disk accretion onto the central star. TW Hya is a relatively low accretor $\left(\sim 10^{-9} \mathrm{M}_{\odot} \mathrm{yr}^{-1}\right.$; Alencar \& Batalha 2002, Herczeg et al. 2004, Ingleby et al. 2013) supporting the continued existence of its gas-disk at a relatively old age; however, accretion must nonetheless proceed. Gammie (1996) found that accretion can be sustained within a layer closer to the disk surface $\left(\Sigma_{g}<\right.$ $100 \mathrm{~g} \mathrm{~cm}^{-2}$, limited by CR penetration) and in this case, estimate an accretion rate of $\dot{M} \sim 10^{-8} \mathrm{M}_{\odot} \mathrm{yr}^{-1}$. Parts of the dead-zone can also potentially become "undead" when reactivated by adjacent turbulent gas (Turner \& Sano 2008), in which case the accretion flow in these regions is reduced compared to the active zone but is not zero. Lesur et al. (2014) found inclusion of the Hall effect in MHD shearing box simulations can support efficient accretion onto the star $\dot{M} \lesssim 10^{-7} \mathrm{M}_{\odot} \mathrm{yr}^{-1}$, even for poorly ionized disks, with laminar flow through the dead zone, supporting the hypothesis that dead-zones may be beneficial to dust growth and planetesimal formation. 

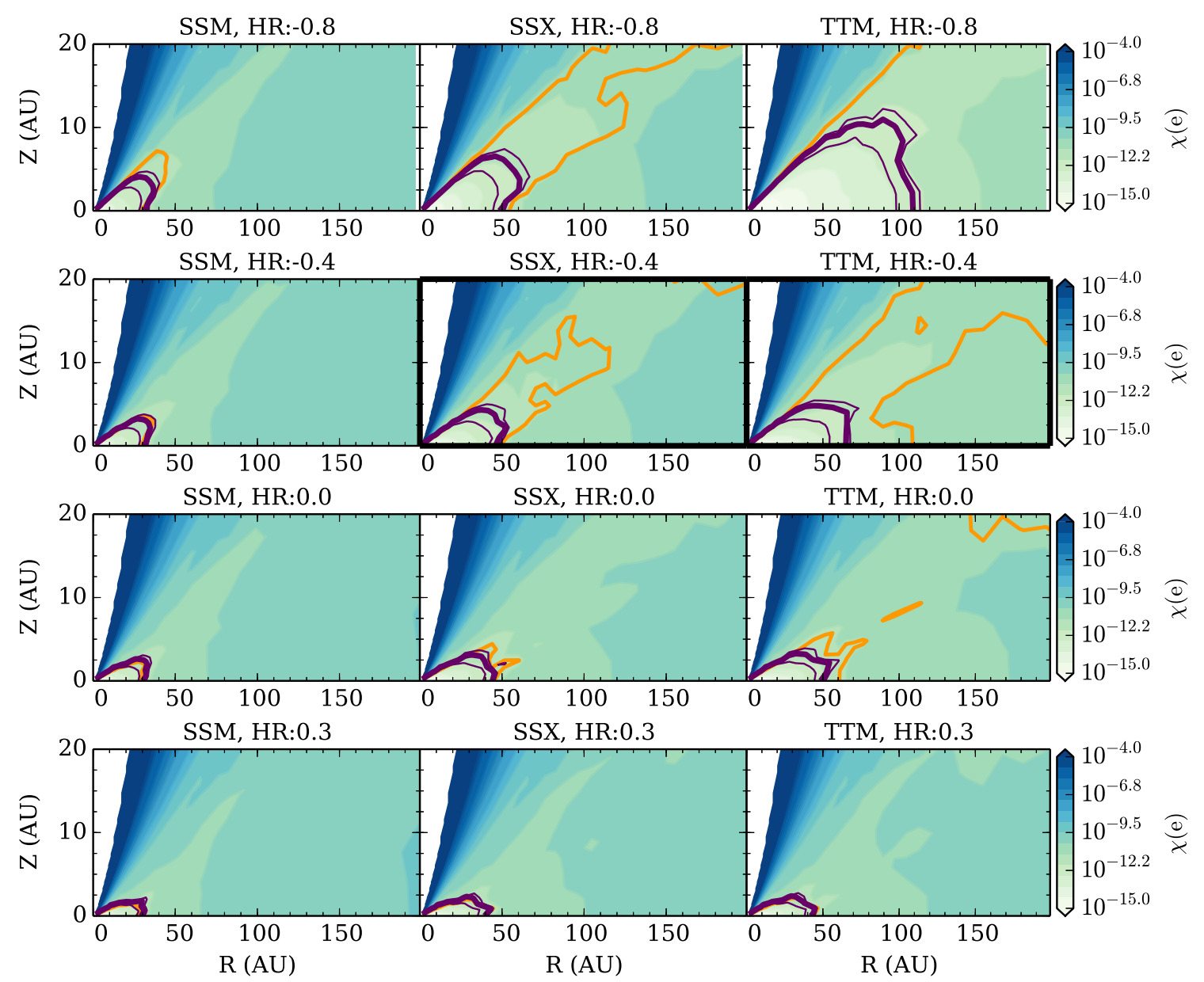

Figure 10. Estimates of the dead-zone size as determined from the chemical calculations (the electron abundances at $1 \mathrm{Myr}$; see Section 3.2. The MRI-dead region is determined from the Reynolds number (Re $<3000$, dark purple) and from the ambipolar diffusion parameter $(A m<0.1$, orange) following Perez-Becker \& Chiang (2011) and Cleeves et al. (2013a). The ambipolar diffusion criteria formally depends upon the strength of the magnetic field, which is unconstrained here, and so we emphasize that the Reynolds number (purple) provides the minimum predicted dead-zone size in this formalism. For the best fit models, the dead-zone extends to $R=50$ AU and $R=65 \mathrm{AU}$ for the SSX and TTM models (black-boxed panels).

Thus the dead-zone may not entirely inhibit disk accretion, though the relationship between massive, extended dead-zones and mass/angular momentum transfer should be studied further.

\section{3. $\mathrm{HCO}^{+}$Asymmetry}

Our new SMA observations reveals a significant smallscale asymmetric emission excess of $\mathrm{HCO}^{+}$. While a detailed analysis of this particular feature is left to future work, there are a few plausible explanations for its origin. The enhancement may be related to a local change in vertical structure, either due to a asymmetric pressure bump or the crest of a spiral arm. The local increase in scaleheight increases the surface area over which the disk can intercept stellar irradiation (Jang-Condell 2008, 2009) including that of X-rays, leading to a local over-production of $\mathrm{HCO}^{+}$. This scenario would also explain why the same feature does not appear in $\mathrm{N}_{2} \mathrm{H}^{+}$, which originates deeper in the disk, hidden from surface irregularities. Alternatively, the presence of an accreting protoplanet still embedded within the disk might locally heat the gas, increasing the local $\mathrm{CO}$ abundance near the midplane and produce deep $\mathrm{HCO}^{+}$; however, the $(3-2)$ transition is likely optically thick and does not directly trace the dense gas where planets are expected to form. The feature may also be temporal in nature, where the same stellar X-ray flares that are known to occur with some frequency may also be related to energetic expulsions from the central star, i.e., coronal mass ejections, that may impinge upon and ionize disk gas directly. All of these scenarios are worth further explanation are beyond the scope of this paper, but hint at interesting chemical structure in the TW Hya disk that should be followed up with high resolution observations.

\section{CONCLUSIONS}

We have constrained the ionization environment of the TW Hya disk using molecular ion observations and a calibrated physical model of the TW Hya dust and gas circumstellar disk, on which we vary the incident ionizing flux of X-rays and CRs. We find that the ionization rate due to CRs is quite low $\left(\zeta_{\mathrm{H}_{2}} \lesssim 10^{-19} \mathrm{~s}^{-1}\right)$, and that the X-ray flares seem to have a lasting chemical effect on the disk. We note that the limit for the $\mathrm{CR}$ rate is more than two orders of magnitude less than that derived for the dense interstellar medium. We emphasize that the particular mechanism via which $\mathrm{CR}$ exclusion happens does not matter, but that the chemistry indicates that 
the CR flux is significantly lower than the canonical values throughout the full radial extent of the disk. The main results of this paper are summarized as follows:

1. The total outer disk ionization rate in the disk midplane is below $\zeta_{\mathrm{H}_{2}} \lesssim 10^{-19} \mathrm{~s}^{-1}$. This values formally puts limits on the combined CR, SLR and Xray ionization rate throughout the disk midplane. $\mathrm{X}$-rays at the $\zeta_{\mathrm{XR}} \sim 10^{-19} \mathrm{~s}^{-1}$ level likely dominate the inner disk midplane and perhaps the outer disk at $2.3 \times 10^{-20} \mathrm{~s}^{-1}$. Due to the likely dominant contribution from scattered stellar X-rays at the midplane, it will be difficult to measure the CR and SLR ionization rate in the TW Hya disk directly. This limit implies that the $\mathrm{CR}$ ionization rate in the outer disk is at least two orders of magnitude below that of the ISM.

2. The $\mathrm{HCO}^{+}$traces a slightly flared state of TW Hya (HR: -0.4) rather than the quiescent X-ray spectrum (HR: -0.8). Additional detailed modeling of the thermal structure with resolved CO observations will help improve the fit to the optically thick $\mathrm{HCO}^{+}$lines, including the $(1-0)$ rotational transitions.

3. We make the first observational prediction of the dead-zone size, and based on the magnetic Reynolds' number, the expected dead-zone coincides with a region of known large grain concentration in the disk out to $60 \mathrm{AU}$. A dead-zone of this size is consistent with the long lifetime of the gas disk in this system.

4. The $\mathrm{HCO}^{+}(3-2)$ emission reveals slight asymmetry, which alone contributes $\sim 20 \%$ of the overall flux. Resolved observations, where available, are thus extremely helpful when trying to understand the bulk disk properties.

In closing, we note that this study provides the strongest constraints to date on the sources of ionization in protoplanetary disks (at least for those constraints derived from imaging observations of ionized molecular species). A wide variety of ionization sources are thought to contribute, including stellar X-rays, SLRs, CRs, and ionizing radiation from the background star-forming environment. This work shows observationally that CRs can indeed be excluded from disks, as proposed previously on theoretical grounds (see, e.g., Cleeves et al. 2013a Padovani et al. 2013, Fatuzzo \& Adams 2014), and provides an estimate for the extent of the dead zone (compare Gammie (1996) with Igea \& Glassgold (1999)). Given the availability of new instruments and facilities, this study marks only the beginning of an important research program that will provide future observational constraints on disk physics.

The authors wish to thank the anonymous referee and editor for their helpful comments. LIC and EAB acknowledge grant AST-1008800 and KO acknowledges support from the Simons Collaboration on the Origins of Life (SCOL) program. CQ acknowledges grant NASA Origins of Solar Systems, Grant Number NNX11AK63G. The Atacama Large Millimeter/submillimeter Array
(ALMA), an international astronomy facility, is a partnership of Europe, North America and East Asia in cooperation with the Republic of Chile. This paper makes use of the following ALMA Science Verification data: ADS/JAO.ALMA\#2011.0.00001.SV.

\section{REFERENCES}

Adams, F. C. 2010, ARA\&A, 48, 47

Adams, F. C., Fatuzzo, M., \& Holden, L. 2014, ApJ, 789, 86

Aikawa, Y., \& Herbst, E. 1999, ApJ, 526, 314

Aikawa, Y., Miyama, S. M., Nakano, T., \& Umebayashi, T. 1996, ApJ, 467, 684

Alencar, S. H. P., \& Batalha, C. 2002, ApJ, 571, 378

Anderson, T. G., Dixon, T. A., Piltch, N. D., et al. 1977, ApJ, 216, L85

Andrews, S. M., Wilner, D. J., Espaillat, C., et al. 2011, ApJ, 732,42

Andrews, S. M., Wilner, D. J., Hughes, A. M., et al. 2012, ApJ, 744,162

Bai, X.-N., \& Stone, J. M. 2011, ApJ, 736, 144

Balbus, S. A., \& Hawley, J. F. 1991, ApJ, 376, 214

Barrado Y Navascués, D. 2006, A\&A, 459, 511

Bergin, E. A., Cleeves, L. I., Crockett, N., \& Blake, G. A. 2014, Faraday Discussions, 168, 61

Bergin, E. A., Cleeves, L. I., Gorti, U., et al. 2013, Nature, 493, 644

Bethell, T. J., \& Bergin, E. A. 2011a, ApJ, 740, 7

- 2011b, ApJ, 739, 78

Birnstiel, T., \& Andrews, S. M. 2014, ApJ, 780, 153

Biver, N., Crovisier, J., Bockelée-Morvan, D., et al. 2012, A\&A, 539, A68

Bockelée-Morvan, D., Crovisier, J., Mumma, M. J., \& Weaver, H. A. 2004, The composition of cometary volatiles, ed. G. W. Kronk (University of Arizona Press), 391-423

Botschwina, P., Horn, M., Flugge, J., \& Seeger, S. 1993, J. Chem. Soc., Faraday Trans., 89, 2219

Brickhouse, N. S., Cranmer, S. R., Dupree, A. K., Luna, G. J. M., \& Wolk, S. 2010, ApJ, 710, 1835

Brinch, C., \& Hogerheijde, M. R. 2010, A\&A, 523, A25

Bruderer, S. 2013, A\&A, 559, A46

Bruderer, S., van Dishoeck, E. F., Doty, S. D., \& Herczeg, G. J. 2012, A\&A, 541, A91

Calvet, N., D'Alessio, P., Hartmann, L., et al. 2002, ApJ, 568, 1008

Calvet, N., \& Gullbring, E. 1998, ApJ, 509, 802

Cleeves, L. I., Adams, F. C., \& Bergin, E. A. 2013a, ApJ, 772, 5

Cleeves, L. I., Adams, F. C., Bergin, E. A., \& Visser, R. 2013b, ApJ, 777, 28

Cleeves, L. I., Bergin, E. A., \& Adams, F. C. 2014a, ApJ, 794, 123

Cleeves, L. I., Bergin, E. A., Alexander, C. M. O., et al. 2014b, Science, 345, 1590

Cohen, O., Drake, J. J., \& Kóta, J. 2012, ApJ, 760, 85

Cutri, R. M., Skrutskie, M. F., van Dyk, S., et al. 2003, 2MASS All Sky Catalog of point sources.

Dauphas, N., \& Chaussidon, M. 2011, Annual Review of Earth and Planetary Sciences, 39, 351

Debes, J. H., Jang-Condell, H., Weinberger, A. J., Roberge, A., \& Schneider, G. 2013, ApJ, 771, 45

Dolginov, A. Z., \& Stepinski, T. F. 1994, ApJ, 427, 377

Dullemond, C. P., \& Dominik, C. 2004, A\&A, 421, 1075

Dupree, A. K., Brickhouse, N. S., Cranmer, S. R., et al. 2012, ApJ, 750, 73

Dutrey, A., Henning, T., Guilloteau, S., et al. 2007, A\&A, 464, 615

Fatuzzo, M., \& Adams, F. C. 2014, ApJ, 787, 26

Favre, C., Cleeves, L. I., Bergin, E. A., Qi, C., \& Blake, G. A. 2013, ApJ, 776, L38

Feigelson, E. D. 1996, ApJ, 468, 306

Feigelson, E. D., Broos, P., Gaffney, III, J. A., et al. 2002, ApJ, 574,258

Fleming, T., \& Stone, J. M. 2003, ApJ, 585, 908

Flower, D. R. 1999, MNRAS, 305, 651

Fogel, J. K. J., Bethell, T. J., Bergin, E. A., Calvet, N., \& Semenov, D. 2011, ApJ, 726, 29 
Furlan, E., Hartmann, L., Calvet, N., et al. 2006, ApJS, 165, 568 Gammie, C. F. 1996, ApJ, 457, 355

Garrod, R. T., Weaver, S. L. W., \& Herbst, E. 2008, ApJ, 682, 283

Glassgold, A. E., Galli, D., \& Padovani, M. 2012, ApJ, 756, 157

Glassgold, A. E., Najita, J., \& Igea, J. 1997, ApJ, 480, 344

—. 2004, ApJ, 615, 972

Gorti, U., \& Hollenbach, D. 2009, ApJ, 690, 1539

Gorti, U., Hollenbach, D., Najita, J., \& Pascucci, I. 2011, ApJ, 735,90

Gounelle, M., Shu, F. H., Shang, H., et al. 2001, ApJ, 548, 1051

Gressel, O., Nelson, R. P., \& Turner, N. J. 2012, MNRAS, 422, 1140

Güdel, M., \& Telleschi, A. 2007, A\&A, 474, L25

Harries, T. J. 2000, MNRAS, 315, 722

Harries, T. J., Monnier, J. D., Symington, N. H., \& Kurosawa, R. 2004, MNRAS, 350, 565

Hartmann, L., Megeath, S. T., Allen, L., et al. 2005, ApJ, 629, 881

Hasegawa, T. I., Herbst, E., \& Leung, C. M. 1992, ApJS, 82, 167

Henkel, C., Wilson, T. L., Langer, N., Chin, Y.-N., \& Mauersberger, R. 1994, in Lecture Notes in Physics, Berlin Springer Verlag, Vol. 439, The Structure and Content of Molecular Clouds, ed. T. L. Wilson \& K. J. Johnston, 72-88

Herbst, E., \& Klemperer, W. 1973, ApJ, 185, 505

Herczeg, G. J., Linsky, J. L., Valenti, J. A., Johns-Krull, C. M., \& Wood, B. E. 2002, ApJ, 572, 310

Herczeg, G. J., Wood, B. E., Linsky, J. L., Valenti, J. A., \& Johns-Krull, C. M. 2004, ApJ, 607, 369

Ho, P. T. P., Moran, J. M., \& Lo, K. Y. 2004, ApJ, 616, L1

Hoff, W., Henning, T., \& Pfau, W. 1998, A\&A, 336, 242

Hogerheijde, M. R., Bergin, E. A., Brinch, C., et al. 2011, Science, 334, 338

Hughes, A. M., Wilner, D. J., Andrews, S. M., Qi, C., \& Hogerheijde, M. R. 2011, ApJ, 727, 85

Igea, J., \& Glassgold, A. E. 1999, ApJ, 518, 848

Indriolo, N., \& McCall, B. J. 2012, ApJ, 745, 91

Ingleby, L., Calvet, N., Herczeg, G., et al. 2013, ApJ, 767, 112

Isella, A., Carpenter, J. M., \& Sargent, A. I. 2009, ApJ, 701, 260

Jang-Condell, H. 2008, ApJ, 679, 797

—. 2009, ApJ, 700, 820

Jonkheid, B., Faas, F. G. A., van Zadelhoff, G.-J., \& van Dishoeck, E. F. 2004, A\&A, 428, 511

Kamp, I., \& Dullemond, C. P. 2004, ApJ, 615, 991

Kastner, J. H., Huenemoerder, D. P., Schulz, N. S., Canizares, C. R., \& Weintraub, D. A. 2002, ApJ, 567, 434

Kastner, J. H., Huenemoerder, D. P., Schulz, N. S., \& Weintraub, D. A. 1999, ApJ, 525, 837

Kastner, J. H., Zuckerman, B., Weintraub, D. A., \& Forveille, T. 1997, Science, 277, 67

Krist, J. E., Stapelfeldt, K. R., Ménard, F., Padgett, D. L., \& Burrows, C. J. 2000, ApJ, 538, 793

Kurosawa, R., Harries, T. J., Bate, M. R., \& Symington, N. H. 2004, MNRAS, 351, 1134

Lamzin, S. A. 1999, Astronomy Letters, 25, 430

Lesur, G., Kunz, M. W., \& Fromang, S. 2014, A\&A, 566, A56

Linsky, J. L. 1998, Space Sci. Rev., 84, 285

Low, F. J., Smith, P. S., Werner, M., et al. 2005, ApJ, 631, 1170

Mathis, J. S., Rumpl, W., \& Nordsieck, K. H. 1977, ApJ, 217, 425

Matsumura, S., \& Pudritz, R. E. 2005, ApJ, 618, L137

Matsumura, S., Pudritz, R. E., \& Thommes, E. W. 2007, ApJ, 660,1609

Mekkaden, M. V. 1998, A\&A, 340, 135

Mentuch, E., Brandeker, A., van Kerkwijk, M. H., Jayawardhana, R., \& Hauschildt, P. H. 2008, ApJ, 689, 1127

Menu, J., van Boekel, R., Henning, T., et al. 2014, A\&A, 564, A93

Moskalenko, I. V., Strong, A. W., Ormes, J. F., \& Potgieter, M. S. 2002, ApJ, 565, 280

Öberg, K. I., Boogert, A. C. A., Pontoppidan, K. M., et al. 2011a, ApJ, 740, 109

Öberg, K. I., Qi, C., Wilner, D. J., \& Andrews, S. M. 2011b, ApJ, 743,152
Öberg, K. I., Qi, C., Wilner, D. J., \& Hogerheijde, M. R. 2012, ApJ, 749, 162

Ouellette, N., Desch, S. J., \& Hester, J. J. 2007, ApJ, 662, 1268 —. 2010, ApJ, 711, 597

Padovani, M., Hennebelle, P., \& Galli, D. 2013, A\&A, 560, A114

Perez-Becker, D., \& Chiang, E. 2011, ApJ, 727, 2

Pinte, C., Harries, T. J., Min, M., et al. 2009, A\&A, 498, 967

Prantzos, N., Aubert, O., \& Audouze, J. 1996, A\&A, 309, 760

Qi, C., Kessler, J. E., Koerner, D. W., Sargent, A. I., \& Blake, G. A. 2003, ApJ, 597, 986

Qi, C., Öberg, K. I., \& Wilner, D. J. 2013a, ApJ, 765, 34

Qi, C., Wilner, D. J., Aikawa, Y., Blake, G. A., \& Hogerheijde, M. R. 2008, ApJ, 681, 1396

Qi, C., Ho, P. T. P., Wilner, D. J., et al. 2004, ApJ, 616, L11

Qi, C., Öberg, K. I., Wilner, D. J., et al. 2013b, Science, 341, 630

Raassen, A. J. J. 2009, A\&A, 505, 755

Roberge, A., Weinberger, A. J., \& Malumuth, E. M. 2005, ApJ, 622,1171

Rucinski, S. M., \& Krautter, J. 1983, A\&A, 121, 217

Sano, T., Miyama, S. M., Umebayashi, T., \& Nakano, T. 2000, ApJ, 543, 486

Sano, T., \& Stone, J. M. 2002, ApJ, 577, 534

Sastry, K. V. L. N., Helminger, P., Herbst, E., \& De Lucia, F. C. 1981, Chemical Physics Letters, 84, 286

Schöier, F. L., van der Tak, F. F. S., van Dishoeck, E. F., \& Black, J. H. 2005, A\&A, 432, 369

Schwarz, K. R., \& Bergin, E. A. 2014, ArXiv e-prints, arXiv:1411.1403

Semenov, D. 2010, ArXiv e-prints, arXiv:1011.4770

Semenov, D., \& Wiebe, D. 2011, ApJS, 196, 25

Semenov, D., Wiebe, D., \& Henning, T. 2004, A\&A, 417, 93

Skinner, S. L., Guedel, M., Koyama, K., \& Yamauchi, S. 1997, ApJ, 486, 886

Smith, I. W. M., Herbst, E., \& Chang, Q. 2004, MNRAS, 350, 323

Stelzer, B., \& Schmitt, J. H. M. M. 2004, A\&A, 418, 687

Stone, J. M., Hawley, J. F., Gammie, C. F., \& Balbus, S. A. 1996, ApJ, 463, 656

Svensmark, H. 2006, Astronomische Nachrichten, 327, 871

Tachihara, K., Neuhäuser, R., \& Fukui, Y. 2009, PASJ, 61, 585

Thi, W.-F., Mathews, G., Ménard, F., et al. 2010, A\&A, 518, L125

Tielens, A. G. G. M., \& Hagen, W. 1982, A\&A, 114, 245

Trilling, D. E., Koerner, D. W., Barnes, J. W., Ftaclas, C., \& Brown, R. H. 2001, ApJ, 552, L151

Turner, N. J., \& Sano, T. 2008, ApJ, 679, L131

Uchida, K. I., Calvet, N., Hartmann, L., et al. 2004, ApJS, 154, 439

Vacca, W. D., \& Sandell, G. 2011, ApJ, 732, 8

van Dishoeck, E. F., Thi, W.-F., \& van Zadelhoff, G.-J. 2003, A\&A, 400, L1

van Leeuwen, F. 2007, A\&A, 474, 653

van Zadelhoff, G.-J., van Dishoeck, E. F., Thi, W.-F., \& Blake,

G. A. 2001, A\&A, 377, 566

Velikhov, E. P. 1959, JETP, 36, 995

Wardle, M. 1999, MNRAS, 307, 849

Webb, R. A., Zuckerman, B., Platais, I., et al. 1999, ApJ, 512, L63

Webber, W. R. 1998, ApJ, 506, 329

Weidenschilling, S. J. 1977, MNRAS, 180, 57

Weinberger, A. J., Anglada-Escudé, G., \& Boss, A. P. 2013, ApJ, 762,118

Weinberger, A. J., Becklin, E. E., Schneider, G., et al. 2002, ApJ, 566,409

Weintraub, D. A., Sandell, G., \& Duncan, W. D. 1989, ApJ, 340, L69

Weintraub, D. A., Saumon, D., Kastner, J. H., \& Forveille, T. 2000, ApJ, 530, 867

Williams, J. P., \& Best, W. M. J. 2014, ApJ, 788, 59

Wilner, D. J., Bourke, T. L., Wright, C. M., et al. 2003, ApJ, 596, 597

Wilner, D. J., D'Alessio, P., Calvet, N., Claussen, M. J., \& Hartmann, L. 2005, ApJ, 626, L109

Wilner, D. J., Ho, P. T. P., Kastner, J. H., \& Rodríguez, L. F. 2000, ApJ, 534, L101

Yan, M., Sadeghpour, H. R., \& Dalgarno, A. 1998, ApJ, 496, 1044 


\section{APPENDIX}

\section{A. PHYSICAL STRUCTURE}

\section{A.1. Dust Model}

We calibrate the disk physical density and temperature structure by fitting TW Hya's spectral energy distribution (SED). References for the SED photometry are provided in the Figure 12 caption, originally compiled by Andrews et al. (2012). We adopt the same parametric density profile presented in Cleeves et al. (2013a) Eqs. (1-4), adapted from Andrews et al. (2011). In essence, the gas and dust surface densities, $\Sigma_{g, d}$, are described by radial power-laws with an exponential taper, while the density, $\rho_{g, d}$, is taken to be vertically Gaussian. Moreover, we break the dust into two populations, one of small "atmosphere" grains with radii $r_{g}=0.005-1 \mu \mathrm{m}$ distributed over the full (gas) scale height of the disk, and a second of larger midplane grains, $r_{g}=0.005-1 \mathrm{~mm}$, concentrated near the midplane. The former contains $10 \%$ of the total dust mass, while the latter contains the remaining 90\%. This larger population is designed to simulate the effects of settling due to grain-growth, a feature common of observed protoplanetary disks (Furlan et al. 2006). Both large and small dust populations have an MRN size distribution (Mathis et al. 1977), where

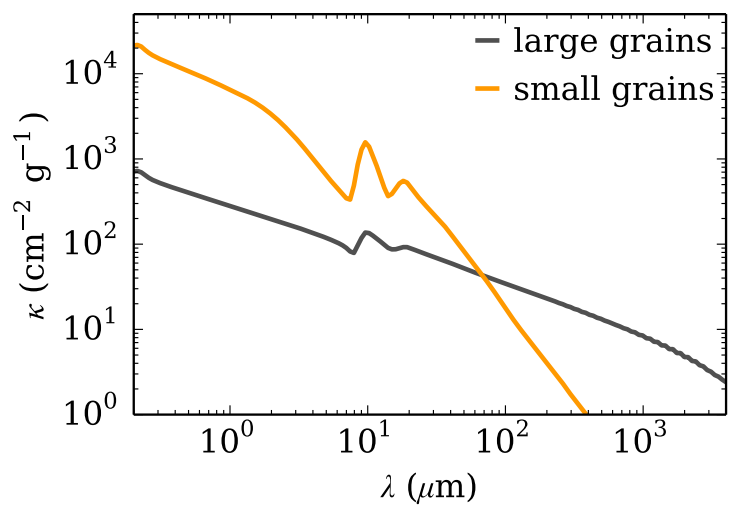

Figure 11. D

ust opacities for the two dust populations used in our SED model. Small grains (atmosphere) have a maximum grain size of $1 \mu$ m, while the large grain population has a maximum wavelength of $1 \mathrm{~mm}$. Opacities are plotted as a cross section per unit dust mass in grams.

the number of grains scales with the size of grain as $n_{g} \propto r_{g}^{-3.5}$. We assume a mixed dust composition with $80 \%$ astronomical silicates and $20 \%$ graphite by mass. The model opacities are shown in Figure 11. Similar to Eqs. (1-4) of Cleeves et al. (2013a), our best fit model has a total dust surface density of

$$
\Sigma_{d}(R)=0.04 \mathrm{~g} \mathrm{~cm}^{-2}\left(\frac{R}{R_{c}}\right)^{-1} \exp \left[-\frac{R}{R_{c}}\right],
$$

and a scale height for small grains (and gas) following

$$
h(R)=15 \mathrm{AU}\left(\frac{R}{R_{c}}\right)^{0.3},
$$

where the critical radius is $R_{c}=150 \mathrm{AU}$. The density of the small and large grain populations are described by

$$
\rho_{s}(R, Z)=\frac{(1-f) \Sigma_{d}}{\sqrt{2 \pi} R h} \exp \left[-\frac{1}{2}\left(\frac{Z}{h}\right)^{2}\right],
$$

and

$$
\rho_{l}(R, Z)=\frac{f \Sigma_{d}}{\sqrt{2 \pi} R \chi h} \exp \left[-\frac{1}{2}\left(\frac{Z}{\chi h}\right)^{2}\right],
$$

respectively, where the fraction of mass in large grains is $f=0.9$ and the large grains concentrated over $\chi=0.2$ the scale height of the small grains, $h . Z$ corresponds to the vertical distance from the midplane where $R$ and $Z$ are in cylindrical coordinates.

We use the radiative transfer code TORUS (Harries 2000, Harries et al. 2004, Kurosawa et al. 2004, Pinte et al. 2009 ) to calculate the dust temperatures and emergent SED assuming dust radiative equilibrium where heating is dominated by the central star. We adopt the following stellar parameters for TW Hya: $T_{\text {eff }}=4110 \mathrm{~K}, M_{*}=0.8 \mathrm{M}_{\odot}$, 


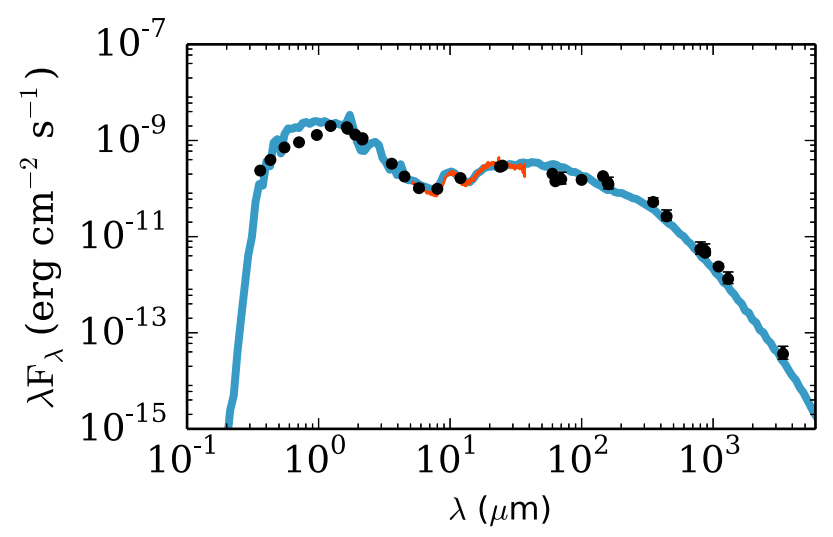

Figure 12. Our best fit spectral energy distribution (blue line) from the combined star and disk of TW Hya. Black points (with error bars) are individual photometric measurements taken from the literature (Weintraub et al. 2000, Mekkaden 1998 Cutri et al. 2003 Hartmann

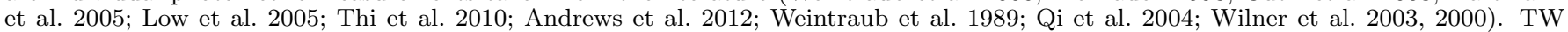
Hya's Spitzer IRS spectrum is over-plotted in orange (Uchida et al. 2004).

$R_{*}=1.04 \mathrm{R}_{\odot}$ (Andrews et al. 2012). The total mass of dust with grain sizes up to $1 \mathrm{~mm}$ in our best fit model is $M_{d}=4 \times 10^{-4} \mathrm{M}_{\odot}$. There may certainly be larger "pebbles," boulders, or even planetesimals; however, the SED modeling is not sensitive to these. We present the best fit SED model in Figure 12. The corresponding dust density and temperature models are shown as solid contour lines in Figures $3 \mathrm{a}$ and 3 b.

\section{A.2. Gas Model}

The SED fitting formally constrains the distribution of dust grains. To constrain the total gas mass in the TW Hya protoplanetary disk, we use the Herschel detection of hydrogen deuteride, HD, to directly probe the gas reservoir (Bergin et al. 2013). This is especially crucial for TW Hya's disk, as the more widely used molecular gas probe, CO, is measured to be depleted in warm gas, i.e., in addition to CO freeze-out (Favre et al. 2013, see also Sec. B.1). Because $\mathrm{HD}$ does not freeze-out and the $\mathrm{HD}$ to $\mathrm{H}_{2}$ ratio is approximately constant and well constrained within the local bubble $\left(\mathrm{HD} / \mathrm{H}_{2}=3 \pm 0.2 \times 10^{-5}\right.$; Linsky 1998$)$, HD is a chemically "simple" species, differing mainly from $\mathrm{H}_{2}$ with regards to UV self-shielding.

To calibrate our model's gas mass using HD, we start from the dust-derived physical structure and assume the gas density and small grains are distributed over the same scale height and are vertically Gaussian (filled contours in Fig. 3a). Furthermore, we must assume a gas-to-dust mass conversion factor where, because gas and dust are not uniformly vertically distributed due to settling, we compare the vertically integrated quantities, $f_{g}=\Sigma_{g} / \Sigma_{d}$, with $f_{g}=100$ for dense interstellar gas.

To calculate the HD emission, we must also estimate the gas temperature and dust opacity at $112 \mu \mathrm{m}$. HD is particularly sensitive to the gas temperature due to the high upper state energy of the $J=1$ rotational level. In the upper layers of the disk, the gas temperature can exceed the dust temperature, i.e., become "decoupled" due to less efficient gas cooling. We estimate the gas temperature by using a fitting function calibrated to thermochemical models of FUV heating from the central star (S. Bruderer, in private communication 2013). Based on a grid of physical structures and FUV field strengths, the detailed heating and cooling balance was solved (e.g., Bruderer 2013) to determine the gas thermal decoupling from the dust in the disk atmosphere where $A_{V}=1-3$. Based on these models, S. Bruderer estimates a parameterized fit to the gas temperature based on the local FUV strength and gas density. We emphasize that the main results of this paper, the ions, are not sensitive to the gas temperatures as most of the molecular ion emission comes from deeper layers and, correspondingly, higher $A_{V}$. To determine the FUV radiation field throughout the disk, we use a Monte Carlo technique to calculate the wavelength-dependent UV field including both absorption and scattering on dust, in addition to the transfer of Lyman- $\alpha$ photons (Bethell \& Bergin 2011b). Special treatment of Lyman- $\alpha$ radiative transfer is important as these photons will resonantly scatter on atomic hydrogen atoms, greatly enhancing the scattered radiation field deep in the disk compared to the primarily forward-scattering dust. Furthermore, Lyman- $\alpha$ carries 85\% of the FUV flux below $2000 \AA$ (Herczeg et al. 2004). The UV optical properties are taken from the dust model described in $\$ 5.2$, and we use the measured TW Hya FUV fluxes from Herczeg et al. (2002, 2004) assuming a distance of $d=55 \mathrm{pc}$. The calculated FUV radiation field integrated over wavelength is shown in Figure 3 $\mathrm{d}$, and the resulting gas temperature structure is shown in Figure $3 \mathrm{~b}$ as filled color contours. For the most part $T_{g}=T_{d}$ (see contour lines), however the gas temperature becomes decoupled from the dust by more than $\Delta T>10 \mathrm{~K}$ in the layer where $z / r \gtrsim 0.4$.

In addition to the gas temperature, the HD emission is sensitive to the vertical structure of the dust as the dust disk becomes optically thick at $112 \mu \mathrm{m}$. Consequently, the HD emission is sensitive to the specific dust opacity for which we must assume a single value. The weighted average of the two large and small populations corresponds to an opacity per gram of dust of $\kappa_{\text {mix }}(112 \mu \mathrm{m})=30 \mathrm{~cm}^{2} \mathrm{~g}^{-1}$.

From the gas and dust density and temperature model, we compute the baseline chemistry for HD (see details of the chemical code in $\$ 3.2$ to primarily determine how much HD is dissociated in the upper layers before self-shielding 
takes hold. Because HD does not freeze-out, the HD abundance is effectively constant throughout the disk below the UV self shielding layer $(z / r \sim 0.4)$. From the calculated HD abundances, we then compute the emergent HD $(1-0)$ line intensity assuming the emission is in LTE (see $\$ 3.4$ for details on the radiative transfer). We then adjust the gas-to-dust ratio, $f_{g}$, until we find agreement with the observed HD flux, $\int F_{\mathrm{HD}} d v=70.6 \pm 7.8 \mathrm{Jy} \mathrm{km} \mathrm{s}^{-1}$ (Bergin et al. 2013). With a vertically integrated gas-to-dust mass ratio of $f_{g}=\Sigma_{g} / \Sigma_{d}=75-100\left(M_{g}=0.03-0.04 \mathrm{M}_{\odot}\right)$, we find good agreement with the observed value, where our $0.04 \mathrm{M}_{\odot}$ model predicts $\int F_{\mathrm{HD}} d v=76.6 \mathrm{Jy} \mathrm{km} \mathrm{s}{ }^{-1}$. We note, however, that slightly less massive $\left(M_{g}=0.02 \mathrm{M}_{\odot}\right)$ but warmer disk or perhaps a more massive $\left(M_{g}=0.05 \mathrm{M}_{\odot}\right)$ but cooler disk can also reproduce the observations, so in the present framework, we find that TW Hya's gas mass is $M_{g}=0.04 \pm 0.02 \mathrm{M}_{\odot}$. This value can be further refined with better observational constraints on the overall vertical density and thermal structure. The gas mass derived here is slightly less than the mass provided by Bergin et al. (2013), $M_{g}>0.05 \mathrm{M}_{\odot}$, and is chiefly due to differences in the gas temperature calculation and underlying disk model assumed.

\section{B. NEUTRAL GAS CONSTRAINTS}

\section{B.1. Neutral Gas Constraints: $C O$}

In the ISM, $\mathrm{CO}$ is the second-most abundant gas phase molecule after $\mathrm{H}_{2}$. In the ISM, $\mathrm{CO}$ has an abundance of $\chi(\mathrm{CO})=10^{-4}$ and participates in a wide range of chemical reactions. However, recent observations indicate that $\mathrm{CO}$ is substantially reduced in warm $(T \gtrsim 20 \mathrm{~K})$ gas where the $\mathrm{CO}$ abundance relative to $\mathrm{H}_{2}$ was found to be $\chi(\mathrm{CO})=(1-10) \times 10^{-6}$ (Andrews et al. 2012, Favre et al. 2013). Williams \& Best (2014) indirectly confirm this finding by deriving a gas mass from CO isotopologue observations of $M_{g}=5 \times 10^{-4}$, a factor of $\sim 100$ less than the HD derived gas mass. One potential explanation for this large $\mathrm{CO}$ deficit is through $\mathrm{CO}$ dissociation by $\mathrm{He}^{+}$, where some fraction of the carbon from $\mathrm{CO}$ to be put into other neutral species with higher grain-surface binding energies than that of CO. This process can happen at early stages prior to the formation of the disk, activated by CR ionization, or at later stages in the disk's warm molecular layer initiated by stellar X-ray ionization (Semenov et al. 2004: Bergin et al. 2013). To robustly make predictions for $\mathrm{HCO}^{+}$abundances, which forms from $\mathrm{CO}$ via

$$
\mathrm{H}_{3}^{+}+\mathrm{CO} \rightarrow \mathrm{HCO}^{+}+\mathrm{H}_{2},
$$

we must include the $\mathrm{CO}$ deficit in our model. We initially ran models where the initial (input) $\mathrm{CO}$ abundance is set at $\chi(\mathrm{CO})=1 \times 10^{-6}$, and the rest of the carbon is put into strongly bound, carbon-bearing ices, e.g., methanol. However, even in this instance the carbon in methanol ice was recycled back into gas phase CO in less than $1 \mathrm{Myr}$ in the layers where UV photons are present. Even when we artificially increased the binding energy of methanol, the carbon nevertheless made its way back into gas phase $\mathrm{CO}$, and over-produced the observed $\mathrm{CO}$ emission (i.e., the CO abundance after $1 \mathrm{Myr}$ was far too high to explain the observations).

In the end, we found that the only way to reproduce the low $\mathrm{CO}$ abundance was to reduce the $\mathrm{CO}$ abundance and explicitly not put it into one of the existing network species, thereby net reducing the amount of reactive carbon. The physical interpretation behind this finding is that the carbon no longer in gaseous $\mathrm{CO}$ has gone on to form something similar to macromolecular organic ices. In the presence of UV irradiation, such material is less likely to non-thermally desorb, and are more likely to break up into radical ices, where the products remain on the grains (and are not returned to the gas) and are thought to be key to forming important biogenic organic material.

Taking two models with different CR ionization rates (SSX and W98, 3.3.1), we i) vary the initial CO abundance, ii) calculate the final $\mathrm{CO}$ abundance after $1 \mathrm{Myr}$, and iii) compute the ${ }^{13} \mathrm{CO}$ and $\mathrm{C}^{18} \mathrm{O}$ emergent line emission (see 3.4). We compare these values to the observations (Favre et al. 2013, see also Table 1). Figure 13 shows the ratio of the observed flux to the model flux for different $\mathrm{CO}$ initial conditions. The model which simultaneously best fits ${ }^{13} \mathrm{CO}$ and $\mathrm{C}^{18} \mathrm{O}$ is one where $\chi(\mathrm{CO})=1 \times 10^{-6}$, though $\mathrm{CO}$ abundances between $\chi(\mathrm{CO})=(5-20) \times 10^{-7}$ can fit either ${ }^{13} \mathrm{CO}$ or $\mathrm{C}^{18} \mathrm{O}$. The main differences between the present work and Favre et al. (2013) is that we are using a new model and a different method to calculate gas temperature. We confirm the Favre et al. (2013) result that the $\mathrm{CO}$ abundance is substantially lower than the canonical $\chi(\mathrm{CO})=10^{-4}$ in the warm gas by approximately a factor of $\sim 100$.

\section{B.2. Neutral Gas Constraints: HCN}

Motivated by the work of Schwarz \& Bergin (2014, submitted), the initial abundances of nitrogen also play an important role in the chemical outcome of nitrogen bearing species, including $\mathrm{HCN}, \mathrm{NH}_{3}$ and $\mathrm{N}_{2} \mathrm{H}^{+}$. Because $\mathrm{N}_{2} \mathrm{H}^{+}$ is simultaneously affected by ionization and $\mathrm{CO}$ abundance, the neutral species provide a cleaner test of the initial nitrogen abundances in this model. Described in more detail in Schwarz \& Bergin (2014, submitted), the final nitrogen molecular abundances are most sensitive to the following broad groupings of initial nitrogen reservoirs, namely the amount of $\mathrm{NH}_{3}$ ice, $\mathrm{N}_{2}$, and $\mathrm{N}$ and/or single- $\mathrm{N}$ containing molecules. The species $\mathrm{HCN}$ has been previously detected in the TW Hya disk, and thus we use this molecule as a probe of the initial nitrogen portioning in the disk; however, we note that this is only one line and that future more detailed modeling and additional observations will greatly help but additional constraints on the nitrogen assay in disks.

In the midplane, since we use a static disk model, the chemistry is such that the abundance of $\mathrm{NH}_{3}$ ice in the midplane is often very similar to that which was assumed initially, i.e., the ices are not rapidly reprocessed. In this light, we can look to cometary $\mathrm{NH}_{3} / \mathrm{H}_{2} \mathrm{O}$ ratios to put an upper limit on the $\mathrm{NH}_{3}$ ice abundance. Typical ammonia 


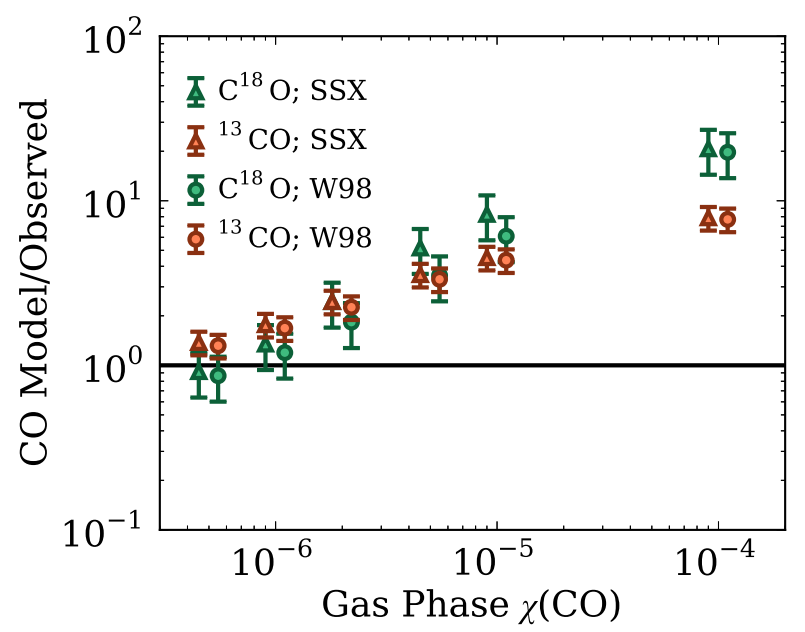

Figure 13. Comparison between the simulated $\mathrm{C}^{18} \mathrm{O}(2-1)$ and ${ }^{13} \mathrm{CO}(2-1)$ line intensities and the observed values (see Table 1 for different gas-phase $\mathrm{CO}$ abundances. The canonical $\mathrm{CO}$ abundance, $\chi(\mathrm{CO})=10^{-4}$ over predicts the observed fluxes by approximately an order of magnitude and is also optically thick - inconsistent with the observations. A gas-phase $\mathrm{CO}$ abundance of $\chi(\mathrm{CO})=10^{-6}$ or perhaps lower is a better match to the data, in agreement with the findings of Andrews et al. (2012) and Favre et al. (2013).

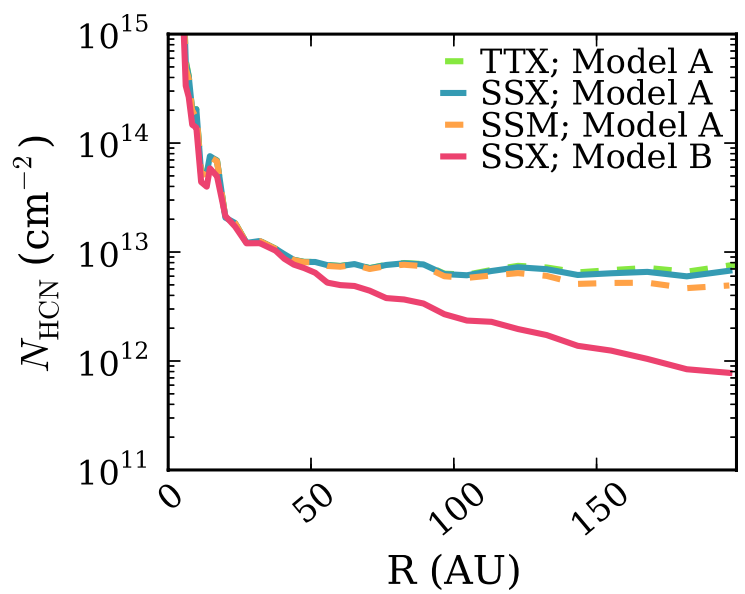

Figure 14. The dependence on different initial nitrogen conditions of the HCN abundance after 1 Myr of chemical evolution. The initial abundances relative to total $\mathrm{H}$ of major nitrogen-bearing species (with abundance $\chi \geq 10^{-7}$ ) are as follows. Model $\mathrm{A}: \chi\left(\mathrm{NH}_{3}\right.$ ice $)=9.16 \times 10^{-6}, \chi\left(\mathrm{N}_{2}\right)=2.5 \times 10^{-6}$, and $\chi(\mathrm{N})=5.1 \times 10^{-6}$. Model B: $\chi\left(\mathrm{NH}_{3}\right.$ ice $)=7.91 \times 10^{-6}, \chi\left(\mathrm{N}_{2}\right)=5.7 \times 10^{-6}$, and $\chi(\mathrm{N})=1 \times 10^{-7}$. Variations between initial nitrogen abundances exceed variations due to different assumed ionization rates (dotted lines, Model A). See Section B.2 for details.

abundances for comets have percentages of $0.1-1.5 \%$ relative to water (Bockelée-Morvan et al.|2004: Biver et al.|2012). Evidence from protostellar $\mathrm{NH}_{3}$ ice abundances, typically $\sim 3 \%$ (Öberg et al. 2011a) and comets gives an approximate range for the amount of nitrogen locked up in ices and thus provides a crude handle on the nitrogen partitioning in the disk. In Figure 14 we show two models considered where Model A has more ammonia ice (less reactive nitrogen)

The initial $\mathrm{N}_{2}$ abundance directly affects $\mathrm{N}_{2} \mathrm{H}^{+}$; however, its effect is predictable. The overall column density profile may shift up or down, but the shape of the $\mathrm{N}_{2} \mathrm{H}^{+}$column density versus radius stays the same. The $\mathrm{N}_{2}$ and $\mathrm{NH}_{3}$ binding energies assumed in the model are $1220 \mathrm{~K}$ and $3080 \mathrm{~K}$, respectively. The final nitrogen abundances determined from the HCN emission are listed in Table 3.

\section{MODEL COMPARISON}

It is important to quantify the model dependency of these results. To determine how our results depend on the disk physical structure, we repeat our experiment using the detailed model of Andrews et al. (2012). The Andrews et al. (2012) model fits the dust distribution in detail, and fits the CO $(3-2)$ profile. Because CO $(3-2)$ is thick, the gas model primarily reflects the disk temperature profile versus radius. However, the best fit model in that work found a significantly smaller taper radius for the gas disk, i.e., the critical radius, where $r_{c}=35 \mathrm{AU}$ for the sA model, which could still fit the distributed CO gas out to $200 \mathrm{AU}$. The model in the present work, for comparison, has a taper at $r_{c}=150 \mathrm{AU}$, dropping off instead at the edge of the $\mathrm{CO}$ and scattered light disk.

Consequently, there is a substantial difference in the mass distribution between the two models, and the disk mass 
Table 3

Chemical model initial abundances relative to total number of H-atoms.

\begin{tabular}{llll}
\hline \hline Species & $\chi$ & Species & $\chi$ \\
\hline $\mathrm{H}_{2}$ & $5.00 \times 10^{-1}$ & $\mathrm{H}_{2} \mathrm{O}(\mathrm{gr})$ & $2.50 \times 10^{-4}$ \\
$\mathrm{HDO}(\mathrm{gr})$ & $1.00 \times 10^{-8}$ & $\mathrm{He}$ & $1.40 \times 10^{-1}$ \\
$\mathrm{CN}$ & $6.60 \times 10^{-8}$ & $\mathrm{HCN}$ & $1.00 \times 10^{-8}$ \\
$\mathrm{~N}$ & $5.10 \times 10^{-6}$ & $\mathrm{NH}_{3}(\mathrm{gr})$ & $9.90 \times 10^{-6}$ \\
$\mathrm{~N}_{2}$ b & $1.00 \times 10^{-6}$ & $\mathrm{H}_{3}^{+}$ & $1.00 \times 10^{-8}$ \\
$\mathrm{CS}$ & $4.00 \times 10^{-9}$ & $\mathrm{SO}^{-}$ & $5.00 \times 10^{-9}$ \\
$\mathrm{Si}^{+}$ & $1.00 \times 10^{-11}$ & $\mathrm{~S}^{+}$ & $1.00 \times 10^{-11}$ \\
$\mathrm{Mg}^{+}$ & $1.00 \times 10^{-11}$ & $\mathrm{Fe}^{+}$ & $1.00 \times 10^{-11}$ \\
$\mathrm{C}^{+}$ & $1.00 \times 10^{-9}$ & $\mathrm{CH}_{4}$ & $1.00 \times 10^{-7}$ \\
$\mathrm{Grain}_{\mathrm{C}}$ & $6.00 \times 10^{-12}$ & $\mathrm{CO}^{\mathrm{c}}$ & $1.00 \times 10^{-6}$ \\
$\mathrm{HD}$ & $7.00 \times 10^{-7}$ & $\mathrm{HCO}^{+}$ & $9.00 \times 10^{-9}$ \\
$\mathrm{HD}$ & $1.50 \times 10^{-5}$ & $\mathrm{H}_{2} \mathrm{D}^{+}$ & $1.30 \times 10^{-10}$ \\
$\mathrm{C}_{2} \mathrm{H}$ & $1.00 \times 10^{-10}$ & $\mathrm{D}_{3}^{+}$ & $2.00 \times 10^{-10}$ \\
\hline
\end{tabular}

${ }^{\mathrm{a}} \mathrm{See} \hat{\mathrm{B} .2}$

${ }^{\mathrm{b}} \mathrm{See} \frac{\mathrm{B} .2}{\mathrm{C} .2}$

${ }^{\mathrm{c}}$ See $\overline{\mathrm{B} .1}$

itself (which is an order of magnitude smaller for the Andrews et al. (2012) model). The mass/density difference is most pronounced at the outer disk, at the same radii where $\mathrm{N}_{2} \mathrm{H}^{+}(4-3)$ is dropping off. By comparing the two models, we can test whether or not the $\mathrm{N}_{2} \mathrm{H}^{+}$distribution is a mass effect or an ionization effect. In Figure 15 , we show the normalized $\mathrm{N}_{2} \mathrm{H}^{+}$column density for the outer disk (Fig. 15a) and the column density of the population of $\mathrm{N}_{2} \mathrm{H}^{+}$in the $J=4$ upper state (which is more closely related to the line emissivity). We have normalized the columns because we have not done any additional chemical calibration or mass calibration for the Andrews et al. (2011) model as were done in the main paper, and so there is an overall offset between the two models. From these tests we find that the overall slope of the $\mathrm{N}_{2} \mathrm{H}^{+}$column density and emissive column density agree well with the results of the main paper for the SSX (reduced CR ionization model), and that even with the reduced outer disk mass in the Andrews et al. (2011) model, the $\mathrm{N}_{2} \mathrm{H}^{+}$emission distribution is too flat to explain the observations. This behavior is a natural consequence of the drop in outer disk density, where the loss of mass acts to reduce the recombination efficiency of ions, and thus there is higher fractional abundance of ions, including $\mathrm{N}_{2} \mathrm{H}^{+}$, than in our model, which has higher outer disk recombination due to the higher outer disk mass in the present paper. Thus the $\mathrm{N}_{2} \mathrm{H}^{+}$profile cannot be attributed to a mass effect, and that a reduced CR ionization rate does a better job of explaining the emission distribution for both physical structures.
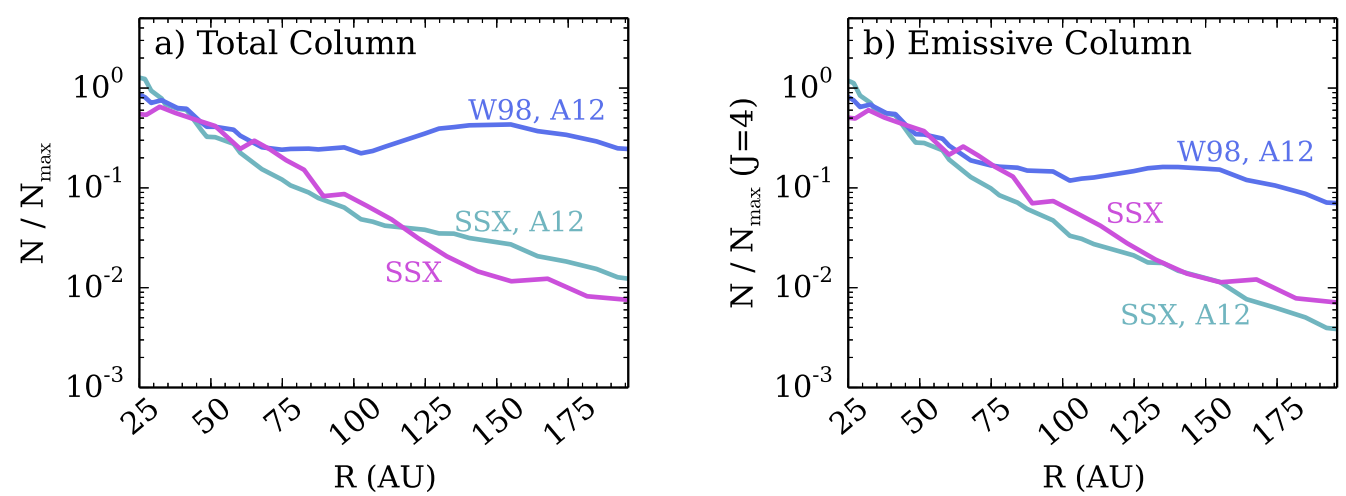

Figure 15. Chemical model comparison between the disk model assumed in this work (labeled just SSX) and the results for a different underlying disk model from Andrews et al. (2012) (lines labeled A12). We find good agreement in the overall slope of the column densities for the low CR ionization model and can exclude the high CR ionization model, W98, which over predicts the outer disk by over an order of magnitude. 